\title{
Allelic variants of OsSUB1A cause differential expression of transcription factor genes in response to submergence in rice
}

Niharika Sharma ${ }^{1}$, Trang Minh Dang ${ }^{2,3}$, Namrata Singh $^{2,4}$, Slobodan Ruzicic $^{5}$, Bernd Mueller-Roeber ${ }^{5}$, Ute Baumann ${ }^{1}$ and Sigrid Heuer ${ }^{1,2,6^{*}}$ (D)

\begin{abstract}
Background: Flooding during seasonal monsoons affects millions of hectares of rice-cultivated areas across Asia. Submerged rice plants die within a week due to lack of oxygen, light and excessive elongation growth to escape the water. Submergence tolerance was first reported in an aus-type rice landrace, FR13A, and the ethylene-responsive transcription factor (TF) gene SUB1A-1 was identified as the major tolerance gene. Intolerant rice varieties generally lack the SUB1A gene but some intermediate tolerant varieties, such as IR64, carry the allelic variant SUB1A-2. Differential effects of the two alleles have so far not been addressed. As a first step, we have therefore quantified and compared the expression of nearly 2500 rice TF genes between IR64 and its derived tolerant near isogenic line IR64-Sub1, which carries the SUB1A-1 allele. Gene expression was studied in internodes, where the main difference in expression between the two alleles was previously shown.

Results: Nineteen and twenty-six TF genes were identified that responded to submergence in IR64 and IR64-Sub1, respectively. Only one gene was found to be submergence-responsive in both, suggesting different regulatory pathways under submergence in the two genotypes. These differentially expressed genes (DEGs) mainly included MYB, NAC, TIFY and Zn-finger TFs, and most genes were downregulated upon submergence. In IR64, but not in IR64-Sub1, SUB1B and SUB1C, which are also present in the Sub1 locus, were identified as submergence responsive. Four TFs were not submergence responsive but exhibited constitutive, genotype-specific differential expression. Most of the identified submergence responsive DEGs are associated with regulatory hormonal pathways, i.e. gibberellins (GA), abscisic acid (ABA), and jasmonic acid (JA), apart from ethylene. An in-silico promoter analysis of the two genotypes revealed the presence of allele-specific single nucleotide polymorphisms, giving rise to ABRE, DRE/CRT, CARE and Site II cis-elements, which can partly explain the observed differential TF gene expression.
\end{abstract}

Conclusion: This study identified new gene targets with the potential to further enhance submergence tolerance in rice and provides insights into novel aspects of SUBTA-mediated tolerance.

Keywords: Submergence tolerance, SUBTA, Rice, Transcription factors

\footnotetext{
* Correspondence: sigrid.heuer@rothamsted.ac.uk

${ }^{1}$ School of Agriculture, Food and Wine, University of Adelaide, Waite

Campus, Plant Genomics Centre, Hartley Grove, Urrbrae, Adelaide, South

Australia 5064, Australia

${ }^{2}$ International Rice Research Institute (IRRI), Los Banos, Philippines

Full list of author information is available at the end of the article
} 


\section{Background}

Rice (Oryza sativa L.) is an important food crop globally and the most advanced genetic monocot model amongst the cereal crops (Cantrell and Reeves, 2002). Biotic and abiotic stresses, such as drought and heat, are known to be detrimental to crop production. Rice production is additionally constraint by submergence stress during the rainy season with complete submergence and water stagnation affecting about 20 million hectares of rice fields in the tropics, causing significant yield and economic losses, and food insecurity (Septiningsih et al., 2012). Rice fields can be flooded with several meters of water for weeks and plants die within a few days from lack of oxygen and impaired photosynthesis (Gibbs and Greenway, 2003; Fukao and Bailey-Serres, 2004). Furthermore, in the attempt to escape the water, plants excessively elongate leaves thereby depleting their carbohydrate and energy reserves, and as water recedes, the plants lodge thus impairing any recovery growth (Singh, Singh, and Ram, 2001; Das, Sarkar, and Ismail, 2005; Fukao, Xu, Ronald, and Bailey-Serres, 2006).

Submergence-tolerant rice varieties were identified in the early 1960s and breeding efforts to introgress this trait into rice cultivars have been ongoing since the 1970s. Submergence tolerance was initially considered a complex trait involving many genes located in multiple quantitative trait loci (QTL) (Mackill, Ismail, Singh, Labios, and Paris, 2012). However, advances in molecular marker technologies and optimized phenotyping techniques aided in the identification of the major QTL Submergence tolerance 1 (Sub1) (Xu, Deb, and Mackill, 2004). Sub1 was identified from the aus-type rice landrace FR13A (Flood Resistance 13A) and it provided a breakthrough in the understanding of submergence tolerance mechanisms, enabling markerassisted breeding of submergence-tolerant rice (Fukao et al., 2006; Xu et al., 2006; Singh, Mackill, and Ismail, 2009; Bailey-Serres et al., 2010; Mackill et al., 2012). Sub1 rice varieties are now widely grown across Asia enhancing plant survival in flooded fields with yield advantages of one ton per hectare or more (Septiningsih et al., 2009; Mackill et al., 2012).

Molecular and comparative sequence analysis of the Sub1 genomic region in submergence-tolerant and -intolerant rice varieties revealed the presence of a variable cluster of two to three ethylene-responsive TFs (ERFs) genes, namely $S U B 1 A, S U B 1 B$ and $S U B 1 C$ (Xu et al., 2006). In submergence-intolerant and moderately tolerant rice genotypes, the $S U B 1 A-1$ gene is either absent, due to an inversion-deletion, or present as an allelic variant, SUB1A-2 (Singh et al., 2010) (Additional file 1: Figure S1a).

Transgenic approaches identified $S U B 1 A-1$ as the major tolerance gene, since its constitutive expression conferred submergence tolerance to an intolerant rice variety (M202), which naturally lacks the SUB1A gene
(Xu et al., 2006). Under complete submergence, the main phenotypic effect observed in SUB1A-1 overexpression and $S u b 1$ near isogenic lines (NILs), is a significantly reduced elongation growth (Additional file 1: Figure S1b). The Sub1 plants assume a "quiescence" status which prevents excessive elongation growth thereby preserving carbohydrate reserves and preventing an energy crisis, as well as lodging once the water recedes (Fukao et al., 2006; Xu et al., 2006; Voesenek and BaileySerres, 2009).

In contrast to the SUB1A-mediated tolerance, deepwater rice responds to submergence with rapid GA-induced elongation growth, so that plants reach the water surface for access to light and oxygen (Fukao et al., 2006; Xu et al., 2006; Bailey-Serres and Voesenek, 2008; Singh et al., 2010). As is the case for $S U B 1 A-1$, rapid elongation growth in deepwater rice is also ethylene induced but regulated by different ERFs, namely SNORKEL1 (SK1) and SNORKEL2 (SK2) (Hattori et al., 2009; Nagai, Hattori, and Ashikari, 2010).

At the molecular level, SUB1A-1 functions by preventing ethylene-induced, GA-mediated elongation growth via enhancing the level of the GA-inhibitors SLENDER RICE 1 (SLR1) and SLENDER RICE LIKE 1 (SLRL1) (Fukao and Bailey-Serres, 2008). Further to this, presence of $S U B 1 A-1$ is associated with the negative regulation of genes involved in carbohydrate catabolism and cell elongation (via expansins) and positive regulation of genes involved in ethanolic fermentation, such as alcohol dehydrogenase (Fukao et al., 2006; Bailey-Serres and Voesenek, 2010). Recently some studies have revealed that $S U B 1 A-1$ is phosphorylated by MITOGEN-ACTIVATED PROTEIN KINASE3 (MAPK3) and in turn physically interacts with MAPK3 and binds to its promoter (Singh and Sinha, 2016). Overall, the suppressed growth and metabolic adjustments during flooding confers a higher recovery rate to Sub1 plants compared to nonSub1 plants as illustrated in the aerial photo of the IRRI demonstration plot (Additional file 1: Figure S1b).

The aforementioned molecular and physiological studies were conducted by comparing Sub1 NILs and SUB1A-1 overexpressing plants with their corresponding wild-type varieties (M202 and Liaogeng, respectively), which have the $S U B 1 B$ and $S U B 1 C$ genes but naturally lack the SUB1A gene (Fukao et al., 2006; Xu et al., 2006). However, an allelic variant of the SUB1A gene exists, $S U B 1 A-2$, which is present in a range of rice varieties (Fukao et al., 2006; Singh et al., 2010). Phenotyping of these $S U B 1 A-2$ varieties revealed a variable level of submergence tolerance ranging from about $4 \%$ to $40 \%$ plant survival after two weeks of submergence. SUB1A expression analysis subsequently showed that both, $S U B 1 A-1$ and $S U B 1 A-2$ are submergenceinducible and expressed at similar levels in leaves, stems 
and developing panicles. However, gene expression in internodes associated well with submergence tolerance, i.e., a higher level of $S U B 1 A$ expression in internodes under submergence was observed in varieties with the Sub1A-1 allele compared to varieties with the SUB1A-2 allele (Singh et al., 2010) (Additional file 1: Figure S1c).

To further address differences between SUB1A-1 and $S U B 1 A-2$ allele effects, we analysed internode samples from IR64 (SUB1A-2) and the IR64-derived NIL IR64Sub1, which carries the tolerant Sub1 locus including the SUB1A-1 allele (Singh et al., 2010). Quantitative real-time PCR (qPCR) targeting nearly 2500 rice TF genes based on the Nipponbare reference genome (Caldana, Scheible, Mueller-Roeber, and Ruzicic, 2007) was conducted with internode samples from plants submerged for $30 \mathrm{~h}$ and corresponding non-submerged control plants to (a) identify TFs that are differentially regulated upon submergence in IR64 and IR64-Sub1, (b) compare TFs in both genotypes to provide insights in the SUB1A regulatory pathway and (c) analyse the promoter region of $S U B 1 A-1$ and $S U B 1 A-2$ to identify single nucleotide polomorphisms (SNPs) that create putative cis-elements and could be binding sites for upstream TFs.

\section{Methods}

\section{Plant material and submergence treatment}

Seeds of the rice variety IR64 (accession number 66970) and the IR64-Sub1 NIL (accession number IR8419422139) were provided by the International Rice Germplasm Collection (IRGC) at IRRI, Philippines. Seeds were incubated at $55{ }^{\circ} \mathrm{C}$ for 5 days to break dormancy before pregermination in petri dishes at $37{ }^{\circ} \mathrm{C}$ in an incubator. Three-day-old seedlings were transplanted into pots filled with soil substituted with $3 \mathrm{~g}$ ammonium sulphate. Four pots with two plants for each accession were grown for 75 days until booting/heading stage, i.e., the onset of internode elongation. At that stage, plants were completely submerged in a concrete tank filled with about $2 \mathrm{~m}$ of tap water for $30 \mathrm{~h}$ (Singh et al., 2010). Nonsubmerged control plants were kept under natural conditions in an IRRI screenhouse and sampled in parallel. From each plant, multiple internodes from three tillers were sampled from a total of four submerged and two control plants per genotype. Samples were immediately frozen in liquid nitrogen and stored at $-80^{\circ} \mathrm{C}$.

\section{RNA isolation and CDNA preparation}

Total RNA was isolated from the internode tissues of the control and submerged plants using TRIzol $^{\odot}$ (Invitrogen) according to the manufacturer's instructions. The quantity and quality of RNA samples were assessed using a Nanodrop ND-100 (Thermo Scientific) and by agarose gel electrophoresis. DNA contamination was removed by treating RNA samples with RNase-free DNase
I according to the protocol provided (Promega). cDNA was synthesized from $5 \mu \mathrm{g}$ of total RNA using Superscript $^{\mathrm{TM}}$ III reverse transcriptase (Invitrogen, Germany) as per the manufacturer's protocol.

\section{Data source for transcription factor sequences and primers}

A qRT-PCR platform was used for expression profiling of the rice TF genes (Caldana et al., 2007). The initial source dataset used for the annotation and primer design was version 2.0 of the Rice Genome Annotation Project (RGAP) (http://rice.plantbiology.msu.edu/) and encompassed a total of 2508 (2487 unique) TF genes. TF sequences were extracted from version 1 of the Plant Transcription Factor Database (Riano-Pachon, Ruzicic, Dreyer, and Mueller-Roeber, 2007; Perez-Rodriguez et al., 2010). The TF genes were initially synchronized with version 5.0 of the RGAP Pseudomolecule and genome annotations resulting in 2221 unique genes with appropriate annotation in version 5.0. Twenty of these were represented in two distinct positions in the qPCR platform as internal controls. Based on version 5.0 of the RGAP Pseudomolecules, 266 unique genes out of 2487 missed annotations (Additional file 2: Table S1). The TF genes were further revised based on the most recent RGAP version 7.0 annotation and details are given in Additional file 2: Table S2. For the genes with missing annotations in Release 7, BLAST searches were performed taking the sequences of the PCR amplicons as queries against the RGAP database.

\section{Transcription factor profiling}

The qPCR platform used for this study had the primer pairs specific to the TF genes described above and were distributed on a total of seven 396 well-plates (Caldana et al., 2007). On each plate, twelve wells were reserved for three reference genes (RGs; represented in quadruplicate) and four wells were for negative water control. For the current study, the RGs 9631.m04973, 9629.m05807 and 9633.m03388 were used (Additional file 2: Table S3 and S4). Of these, 9633.m03388 (LOC_Os05g36290, actin) was selected as the best suitable RG for all subsequent calculations and normalizations. There were three technical replicates for each of the four samples $\left(\right.$ IR64 $_{C}$, IR64 ${ }_{S}$, IR64-Sub1 $1_{C}$ and IR64$\mathrm{Sub}_{\mathrm{S}}$, where $\mathrm{C}$ denoted control and $\mathrm{S}$ submergence treated plants). Real-time PCR analysis was based on SYBR Green (Applied Biosystems, Germany) and was conducted in a total volume of $5 \mu \mathrm{l}(2.5 \mu \mathrm{l}$ of SYBR Green master mix, $0.5 \mu \mathrm{l}$ of cDNA $(1.25 \mathrm{ng} / \mu \mathrm{l})$ and $200 \mathrm{nM}$ forward and reverse primers). PCR amplifications were carried out as described in (Caldana et al., 2007) in an ABI PRISM 7900 HT sequence detection system (Applied Biosystems, Germany). 


\section{Data processing and statistical analysis}

Based on the obtained amplification curves, the $\mathrm{Ct}$ values (fractional cycle number at threshold), the value of PCR efficiency and the corresponding coefficient of determination $\left(\mathrm{R}^{2}\right)$ were calculated using LinRegPCR software (Ruijter et al., 2009) for all four samples (IR64 ${ }_{C}$, $\mathrm{IR}_{\mathrm{S}}$, IR64-Sub1 $\mathrm{C}$ and IR64-Sub1 S $_{\mathrm{S}}$. Ct values for all reactions were $\log _{2}$ transformed (Additional file 2: Table $\mathrm{S} 3$ and S4). The average Ct values for $\mathrm{RGs}\left(\mathrm{Ct}_{\mathrm{RG}}\right)$ were calculated from $\mathrm{Ct}$ values of quadruplicates for each 396 well-plate separately for control and submerged conditions. The RG selected for all subsequent calculations was actin (LOC_Os05g36290). Based on the average $\mathrm{Ct}_{\mathrm{RG}}$, the $\Delta \mathrm{Ct}$ for each selected gene (SG) was calculated as $\Delta \mathrm{Ct}=\mathrm{Ct}_{\mathrm{SG}}-\mathrm{Ct}_{\mathrm{RG}}$. All reactions with $\mathrm{R}^{2}<0.995$, reflecting low-quality amplifications and those designated as "undetermined" (did not deliver a $\mathrm{Ct}<40$ ) were excluded from further analyses. The obtained sets of $\Delta \mathrm{Ct}$ values for control and submerged conditions for each gene model (in three replicates) were examined for significant differential expression using LIMMA's moderated t-test (Smyth, 2005). LIMMA computes moderated $\mathrm{t}$-statistics and log-odds of differential expression by empirical Bayes shrinkage of the standard errors towards a common value. False Discovery Rate (FDR) corrections of $p$-values were carried out using Benjamini and Hochberg method (Benjamini and Hochberg, 1995). A gene was considered differentially expressed if the moderated $\mathrm{t}$-test resulted in a corrected $\mathrm{p}$-value $<0.01$. Hence, the list of differentially expressed TF genes and corresponding $\log _{2} \mathrm{FC}$ (fold change) values under submergence stress were generated from both, the IR64 and the IR64Sub1 submerged plants compared to the respective control plants. In addition, we examined the data for genotype-specific expression differences. For this, we used the contrast matrix referred from the LIMMA manual (https://www.bioconductor.org/packages/devel/ bioc/vignettes/limma/inst/doc/ usersguide.pdf) as follows:

Contrast.matrix <- makeContrasts (SubmergenceIR64 = $\mathrm{IR64}_{\mathrm{S}}$ IR64 $_{\mathrm{C}}, \quad$ SubmergenceIR64Sub1 = IR64Sub1 $1_{\mathrm{S}}$ IR64Sub1 $1_{C}$, Genotype $=\left(I_{\text {IR64Sub1 }}+\right.$ IR64Sub1 $\left._{C}\right)-\left(I R 64_{S}\right.$ $+\mathrm{IR}_{\mathrm{C}}$ ) levels = design).

\section{Promoter analysis}

The promoter sequences of the SUB1A-1 and SUB1A-2 alleles (Additional file 2: Table S5) were aligned using Clustal Omega (http://www.ebi.ac.uk/Tools/msa/clustalo/) and analysed using PlantPAN 2.0 (Chow et al., 2016) as well as manually. A $2 \mathrm{~kb}$ upstream DNA region of the DEGs was extracted from RAP-DB (http:// rapdb.dna.affrc.go.jp/index.html) and analysed by PlantPAN (http://plantpan2.itps.ncku.edu.tw/) to identify putative cis-regulatory elements.

\section{Results}

In order to investigate the difference between submergence tolerance as mediated by the strong allele $S U B 1 A$ 1 (present in IR64-Sub1) and the weak allele SUB1A-2 (present in IR64), expression of 2487 rice TF genes was quantified by qPCR in internodes of submerged and non-submerged control plants. Internodes were chosen for this study since differences in the expression of the SUB1A-1 and SUB1A-2 alleles were most pronounced in this tissue (Singh et al., 2010).

\section{Update of TF gene annotations}

The 2487 TF genes given in Additional file 2: Table S1 were synchronised to the latest version 7.0 of the RGAP Pseudomolecules and annotation of the rice genome (Kawahara et al., 2013). For 72 of the 266 genes with missing annotations, we could obtain annotations from version 7.0 (Additional file 2: Table S2) and one gene was marked as obsolete. Therefore, only 193 genes remained as not being annotated in version 7.0. Nine genes previously annotated in version 5 miss annotations in version 7 , eight of these are now considered obsolete and one is without annotation in versions 6.1 and 7.0 of RGAP annotations. Hence, a total of 2284 TF gene models were updated with version 7.0 annotations (Table 1).

\section{Differential gene expression analysis in IR64 and IR64- Sub1}

The expression patterns of the TF genes in four samples $\left(\right.$ IR64 $_{C}$, IR64 $_{S}$, IR64-Sub1 $1_{C}$ and IR64-Sub1 $1_{S}$ ) were analysed by qPCR. Statistical comparisons for the identification of DEGs were performed only for those genes which possessed at least two calculated $\Delta \mathrm{Ct}$ values in both genotypes and both growth conditions (control and submerged). For performing differential expression analysis an intersect of 1823 TF genes, 1944 genes of the IR64 dataset and 1933 of the IR64-Sub1 dataset, were taken as input for LIMMA's moderated t-test comparisons (Fig. 1a).

Table 1 Summary of the total number of genes with and without annotations in the RGAP pseudomolecules version 7.0

Number of TF Genes with and without annotations in version 7.0 of RGAP annotations

Version 5.0 annotation from RGAP

\begin{tabular}{|c|c|c|c|c|c|}
\hline Genes & All & \multicolumn{2}{|c|}{ Missing annotations } & \multicolumn{2}{|c|}{ With annotations } \\
\hline Total & 2508 & \multicolumn{2}{|l|}{267} & \multicolumn{2}{|l|}{2241} \\
\hline Unique & 2487 & \multicolumn{2}{|l|}{266} & \multicolumn{2}{|l|}{2221} \\
\hline \multicolumn{6}{|c|}{ Version 7.0 annotation from RGAP } \\
\hline & & Obsolete & $\begin{array}{l}\text { With } \\
\text { annotations }\end{array}$ & Obsolete & $\begin{array}{l}\text { Without } \\
\text { annotations }\end{array}$ \\
\hline \multirow[t]{2}{*}{ Unique } & & 1 & 72 & 8 & 1 \\
\hline & & \multicolumn{2}{|c|}{193 missing annotations } & \multicolumn{2}{|c|}{2212 annotated } \\
\hline Total & & & & \multicolumn{2}{|c|}{2284 annotated genes } \\
\hline
\end{tabular}



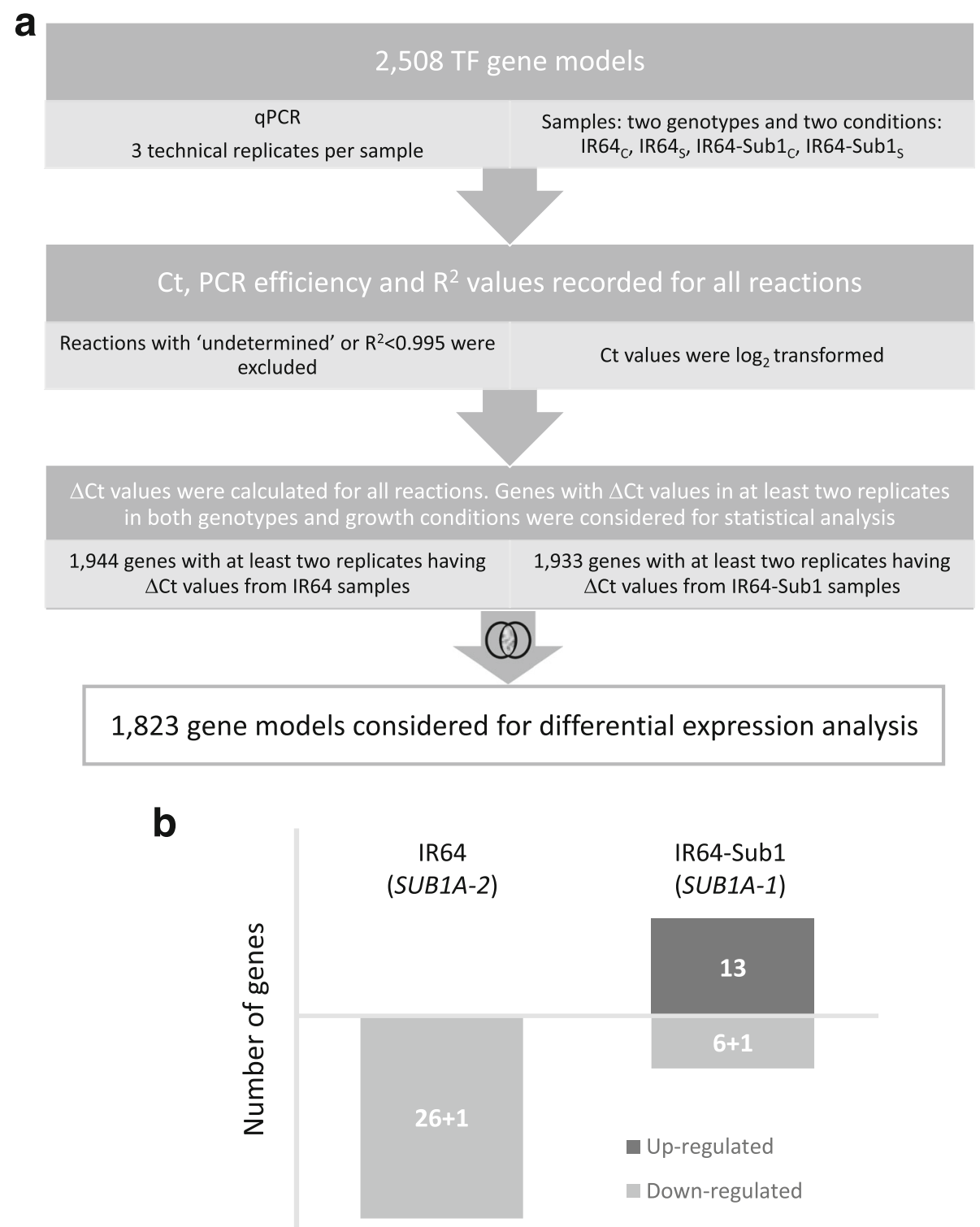

Fig. 1 Identification of differentially expressed transcription factor genes. a Overview of the data processing and selection of genes as input for the statistical analysis of differential gene expression using LIMMA. b Differentially expressed transcription factor genes under submergence stress in IR64 and IR64-Sub1. +1 indicates the common TF gene (MYB), which is submergence-responsive in both genotypes

The resulting $p$-values, FDR corrected $\mathrm{p}$-values and $\log _{2} \mathrm{FC}$ values for all gene models are provided in Additional file 2: Table S6.

\section{Genotype-specific expression differences of TFs}

The genotypic comparison revealed a total of four TF genes that were not submergence responsive but depicted genotype-dependent expression differences between IR64 and IR64-Sub1 (Table 2). These genes code for a basic helixloop-helix (bHLH) factor OsIRO2 (LOC_Os01g72370), OsWRKY21 (LOC_Os01g60640) and two TIFY type TFs (OsTIFY11e/OsZIM18/OsJAZ13 (LOC_Os10g25230); OsTIFY11d/OsJAZ12 (LOC_Os10g25290)). All four genes had overall lower expression levels in IR64-Sub1 compared to IR64 (Fig. 2a).

Studies have shown these four TF genes to be regulated (upregulated $-\uparrow$ or downregulated- $\downarrow$ ) under the following conditions: iron $(\mathrm{Fe})$ starvation (OsIRO2 $\uparrow)$; desiccation/drought (OsIRO2 $\downarrow$, TIFY11d \& 11e $\uparrow)$; salinity (OsIRO2 $\downarrow$, TIFY11d \& 11e $\uparrow)$; salicylic acid (SA) (OsWRKY21 $\uparrow$ ); wounding (TIFY11d \& 11e $\uparrow$ ); pathogens (TIFY11d \& 11e $\uparrow$ ); cold (TIFY11d \& 11e $\uparrow$ ); JA (TIFY11d \& 11e $\uparrow$ ), GA (TIFY11e $\downarrow$ ) and ABA (TIFY11d $\mathcal{E} 11 e \downarrow$ ) (Ogo et al., 2006; Wang et al., 2013; Ramamoorthy, Jiang, Kumar, Venkatesh, and Ramachandran, 2008; Ye, Du, Tang, Li, and Xiong, 2009; Ranjan et al., 
Table 2 Details of the differentially expressed TF genes and their corresponding fold change value (log2FC) under submergence in IR64 and IR64-Sub1

\begin{tabular}{|c|c|c|c|c|c|}
\hline & Gene \# & Locus ID ver 7.0 & Gene Description/TF Family & Gene name & $\mathrm{og} 2 \mathrm{FC}$ \\
\hline \multirow[t]{4}{*}{$\begin{array}{l}\text { Genotype specific } \\
\text { differences }\end{array}$} & 1 & LOC_Os01g72370 & $\begin{array}{l}\text { Helix-loop-helix DNA-binding domain containing } \\
\text { protein; bHLH }\end{array}$ & OsIRO2/OsbHLH056 & -0.64 \\
\hline & 2 & LOC_Os10g25230 & ZIM domain containing protein; Tify & $\begin{array}{l}\text { OsTIFY11e/OsZIM18/ } \\
\text { OsJAZ13 }\end{array}$ & -0.50 \\
\hline & 3 & LOC_Os10g25290 & ZIM domaincontaining protein; Tify & OsTIFY11d/OsJAZ12 & -0.36 \\
\hline & 4 & LOC_Os01g60640 & WRKY21; WRKY & OsWRKY21 & -0.44 \\
\hline \multirow{19}{*}{$\begin{array}{l}\text { Submergence } \\
\text { responsive in IR64 }\end{array}$} & 1 & LOC_Os07g22730 & AP2 domain containing protein; AP2-EREBP & OsERF136/AP2/EREBP\#106 & -0.19 \\
\hline & 2 & LOC_Os05g27930 & AP2 domain containing protein; AP2-EREBP & $\begin{array}{l}\text { OsERF042/AP2/EREBP\#048/ } \\
\text { OsDREB2b }\end{array}$ & -0.28 \\
\hline & 3 & LOC_Os04g47059 & bHLH & OsbHLH16/OSB2 & 0.26 \\
\hline & 4 & LOC_Os01g36220 & $\begin{array}{l}\text { bZIP transcription factor domain containing } \\
\text { protein; bZIP }\end{array}$ & OsbZIP4 & 0.31 \\
\hline & 5 & LOC_Os02g35770 & Homeobox associated leucine zipper; HB & OsHox7 & 0.18 \\
\hline & 6 & LOC_Os02g13800 & $\begin{array}{l}\text { HSF-type DNA binding domain containing } \\
\text { protein; HSF }\end{array}$ & OsHsfC2a/OsHsf-05 & 0.25 \\
\hline & 7 & LOC_Os02g40530 & MYB family transcription factor; MYB & MYB/OsMPS & -0.28 \\
\hline & 8 & LOC_Os07g37210 & MYB family transcription factor; MYB & MYB/OsMyb7 & 0.17 \\
\hline & 9 & LOC_Os11g47460 & MYB family transcription factor; MYB & MYB & 0.16 \\
\hline & 10 & LOC_Os06g51260 & MYB family transcription factor; MYB-related & MYB/ OsLHY-like_chr.6 & 0.36 \\
\hline & 11 & LOC_Os01g47370 & MYB family transcription factor; MYB-related & MYB & 0.20 \\
\hline & 12 & LOC_Os08g06110 & MYB family transcription factor; MYB-related & $\begin{array}{l}\text { MYB/OsCCA1/OsLHY } \\
\text { /LHY-like_chr. } 8\end{array}$ & 0.33 \\
\hline & 13 & LOC_Os07g26150 & MYB family transcription factor; MYB-related & MYB/Hsp40 & 0.18 \\
\hline & 14 & LOC_Os04g41560 & B-box zinc finger family protein; Orphans & OsBBX11/OsSTO & 0.21 \\
\hline & 15 & LOC_Os08g08120 & B-box zinc finger family protein; Orphans & OsBBX24 & 0.21 \\
\hline & 16 & LOC_Os09g25060 & WRKY76; WRKY & OsWRKY76 & -0.31 \\
\hline & 17 & LOC_Os03g08310 & ZIM domain containing protein; Tify & OsTIFY11a/OsJAZ9 & -0.31 \\
\hline & 18 & LOC_Os02g35329 & RING-H2 finger protein ATL3F & ATL3F/OsELF5 & -0.28 \\
\hline & 19 & LOC_Os07g43740 & $\begin{array}{l}\text { Zinc finger, } \mathrm{C} 3 \mathrm{HC} 4 \text { type domain containing } \\
\text { protein }\end{array}$ & Zn finger & 0.20 \\
\hline \multirow[t]{2}{*}{ Common gene } & 1 & LOC_Os01g19330 & MYB family transcription factor; MYB & MYB & -0.394 \\
\hline & & & & & -0.36 \\
\hline \multirow[t]{9}{*}{$\begin{array}{l}\text { Submergence } \\
\text { responsive in IR64 }\end{array}$} & 1 & LOC_Os08g06120 & $\begin{array}{l}\text { B3 DNA binding domain containing protein; } \\
\text { ABI3VP1 }\end{array}$ & Similar to NGA1 (NGATHA1) & -0.26 \\
\hline & 2 & LOC_Os09g11480 & AP2 domain containing protein; AP2-EREBP & $\begin{array}{l}\text { OsSUB1B/OsERF063/AP2/ } \\
\text { EREBP\#166 }\end{array}$ & -0.41 \\
\hline & 3 & LOC_Os09g11460 & AP2 domain containing protein; AP2-EREBP & $\begin{array}{l}\text { OsSUB1C/ OsERF73/AP2/ } \\
\text { EREBP\#122 }\end{array}$ & -0.23 \\
\hline & 4 & LOC_Os07g47790 & AP2 domain containing protein; AP2-EREBP & OsERF067/AP2/EREBP\#076 & -0.22 \\
\hline & 5 & LOC_Os03g22170 & AP2 domain containing protein; AP2-EREBP & OsERF066/AP2/EREBP\#030 & -0.31 \\
\hline & 6 & LOC_Os01g54890 & $\begin{array}{l}\text { Ethylene-responsive transcription factor 2; } \\
\text { AP2-EREBP }\end{array}$ & OsERF922/AP2/EREBP\#078 & -0.21 \\
\hline & 7 & LOC_Os01g64020 & Transcription factor; bZIP & OsLG2/OsbZIP11 & -0.16 \\
\hline & 8 & LOC_Os02g49230 & CCT/B-box zinc finger protein; C2C2-CO-like & OsBBX7 & $\begin{array}{l}-0.17 \\
-0.18 \\
-0.20\end{array}$ \\
\hline & 9 & LOC_Os01g74410 & MYB family transcription factor; MYB & OsMYB48-1 & $\begin{array}{l}-0.23 \\
-0.32\end{array}$ \\
\hline
\end{tabular}


Table 2 Details of the differentially expressed TF genes and their corresponding fold change value (log2FC) under submergence in IR64 and IR64-Sub1 (Continued)

\begin{tabular}{|c|c|c|c|c|}
\hline Gene \# & Locus ID ver 7.0 & Gene Description/TF Family & Gene name & $\mathrm{og} 2 \mathrm{FC}$ \\
\hline 10 & LOC_Os03g20090 & MYB family transcription factor; MYB & OsMYB2 & -0.18 \\
\hline 11 & LOC_Os08g43550 & MYB family transcription factor; MYB & OsMYB7 & -0.24 \\
\hline 12 & LOC_Os03g04900 & MYB family transcription factor; MYB & MYB & -0.20 \\
\hline 13 & LOC_Os11g05614 & No apical meristem protein; NAC & $\begin{array}{l}\text { OsONAC7/ONAC17/ } \\
\text { ONAC30/OsNAC111 }\end{array}$ & -0.26 \\
\hline 14 & LOC_Os07g37920 & No apical meristem protein; NAC & $\begin{array}{l}\text { OsSNAC/ONAC10/ } \\
\text { OsSTA199 }\end{array}$ & -0.18 \\
\hline 15 & LOC_Os03g04070 & No apical meristem protein; NAC & OsANAC34/ONAC22 & -0.21 \\
\hline 16 & LOC_Os01g66120 & No apical meristem protein; NAC & OsNAC6/SNAC2/NAC48 & -0.26 \\
\hline 17 & LOC_Os01g10580 & B-box zinc finger family protein; Orphans & OsBBX1/OsDBB3c & -0.22 \\
\hline 18 & LOC_Os12g10660 & B-box zinc finger family protein; Orphans & OsBBX30 & -0.21 \\
\hline 19 & LOC_Os02g05470 & CCT motif family protein; Orphans & OsCCT03/OsCMF3 & -0.43 \\
\hline 20 & LOC_Os07g07690 & $\begin{array}{l}\text { PHD-finger domain containing protein; } \\
\text { PHD }\end{array}$ & PHD & -0.43 \\
\hline 21 & LOC_Os05g49620 & WRKY19; WRKY & OsWRKY19 & -0.25 \\
\hline 22 & LOC_Os09g25070 & WRKY62; WRKY & OsWRKY62 & -0.33 \\
\hline 23 & LOC_Os02g08440 & WRKY71; WRKY & WRKY71/OsEXB1 & -0.25 \\
\hline 24 & LOC_Os09g30400 & WRKY90; WRKY & OsWRKY90 & -0.18 \\
\hline 25 & LOC_Os06g03580 & Zinc RING finger protein & OsBBI1 & -0.20 \\
\hline 26 & LOC_Os01g11460 & $\begin{array}{l}\text { Zinc finger, } \mathrm{C} 3 \mathrm{HC} 4 \text { type domain containing } \\
\text { protein }\end{array}$ & Zn finger ATL2K & -0.20 \\
\hline
\end{tabular}
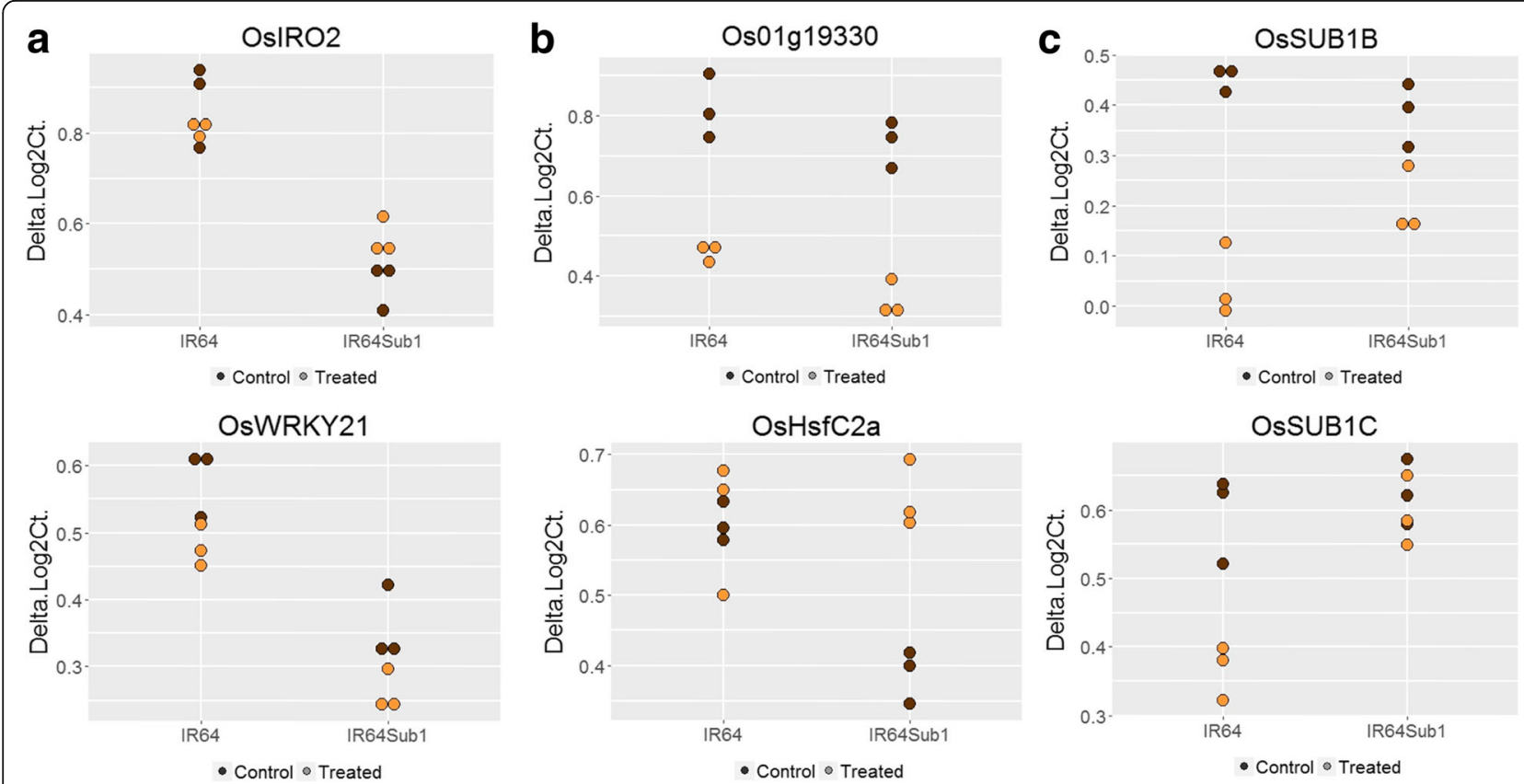

Fig. 2 Differentially expressed transcription factor genes in IR64 and IR64-Sub1. Representative dot plots are shown illustrating three different categories of DEGs: a Non-submergence-responsive genes with genotype-dependent differential expression, (b). submergence-responsive genes in IR64-Sub1, (c). and submergence-responsive genes in IR64 
2015; Yang, Chen, Jen, Liu, and Chang, 2015; Shankar, Bhattacharjee, and Jain, 2016; Xiang et al., 2017). Under submergence, OsTIFY11e was reported to be downregulated in M202-Sub1 compared to M202 but upregulated under drought (Fukao, Yeung, and Bailey-Serres, 2011). Information on these genes is summarized in Figs. 3 and 4 and details are given in Additional file 2: Table S7.

\section{Submergence-responsive TFs in IR64-Sub1}

In IR64-Sub1, 19 TF genes were found to be submergence-responsive, of which thirteen genes were upregulated and six genes were downregulated (Fig. 1b, Table 2). The group of upregulated genes include: 6 MYB factors, 3 Zn finger TFs, 1 Heat Shock Factor (HSF), 1 bHLH, 1 bZIP, and 1 HOX TFs. And the seven $(6+1)$ downregulated genes include: 2 AP2-type TFs, 2 MYBs, a WRKY, a TIFY and a RING-H2 finger gene. One of the seven downregulated genes, the MYB factor LOC_01g19330, was downregulated in response to $30 \mathrm{~h}$ of submergence in both IR64 and IR64-Sub1 genotypes and is placed in the category of a common gene (Fig. 2, Table 2). Studies of these TFs in relation to abiotic stress and hormonal response are detailed below and an overview of the data is provided in Fig. 3.

\section{Upregulated genes}

MYB TFs have been reported to play various biological functions in plants. With respect to abiotic stresses and hormonal pathways the following have been documented in rice: salinity stress (LOC_Os06g51260 $\downarrow$, LOC_Os01g47370 $\downarrow$, LOC_Os07g26150 $\downarrow$ ); GA (MYB_LOC_Os11g47460 $\downarrow$, LOC_Os01g47370 $\downarrow$ ), desiccation/drought showed both up and downregulated depending on variety analysed

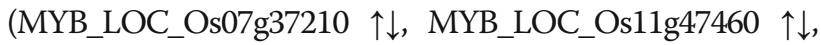

MYB_LOC_Os07g26150 \); and seed dormancy regulated via $\mathrm{ABA}-\mathrm{GA}$ antagonism (MYB_LOC_Os08g06110 $\uparrow$ ) (Baxter et al., 2007; Gao et al., 2009; Katiyar et al., 2012; Boyden et al., 2012; Wu et al., 2016; Park et al., 2015; Shankar et al., 2016; Xiang et al., 2017).

The $\mathrm{Zn}$ finger TFs included two B-Box domain genes, namely OsBBX11 (LOC_Os04g41560) and OsBBX24 (LOC_Os08g08120), and one gene with a Zn-finger binding domain (LOC_Os07g43740). OsBBX11 has been reported to have two BBX domains, whereas OsBBX24 has one BBX domain. These two genes are responsive to various hormones; auxin $(O s B B X 11 \downarrow)$; GA $(O s B B X 11 \downarrow$, OsBBX24 $\downarrow)$; cytokinin $(O s B B X 11 \downarrow)$; kinetin (OsBBX11 $\downarrow, O s B B X 24 \downarrow \downarrow)$ and naphthalene acetic acid (NAA) $(O s B B X 24 \downarrow)$. OsBBX11 was also seen to be upregulated by light and downregulated by desiccation (Huang, Zhao, Weng, Wang, and Xie, 2012; Shankar et al., 2016). LOC_Os07g43740 has been document to be upregulated under several abiotic stresses, i.e. anoxia, drought, desiccation, salt, heat and chilling (Ham, Moon, Hwang, and Jang, 2013; Shankar et al., 2016).

The heat shock factor (HSF) gene, OsHsfC2a (LOC_Os02g13800), which was upregulated upon submergence in IR64-Sub1, has also been reported to have increased expression under other abiotic stresses, i.e. heat, cold, oxidative stress (Mittal et al., 2009; Mittal et al., 2012) as well as drought and desiccation (Chauhan, Khurana, Agarwal, and Khurana, 2011; Shankar et al., 2016). This TF also showed upregulation in response to brassinosteroids (BR) and SA but the expression decreased in response to ABA (Chauhan et al., 2011; Shankar et al., 2016). Promoter analysis of $\mathrm{OsHsfC} 2 a$ revealed presence of light, $\mathrm{ABA}$ and methyl-jasmonate (MeJA) responsive cis-elements (Wang, Zhang, and Shou, 2009).

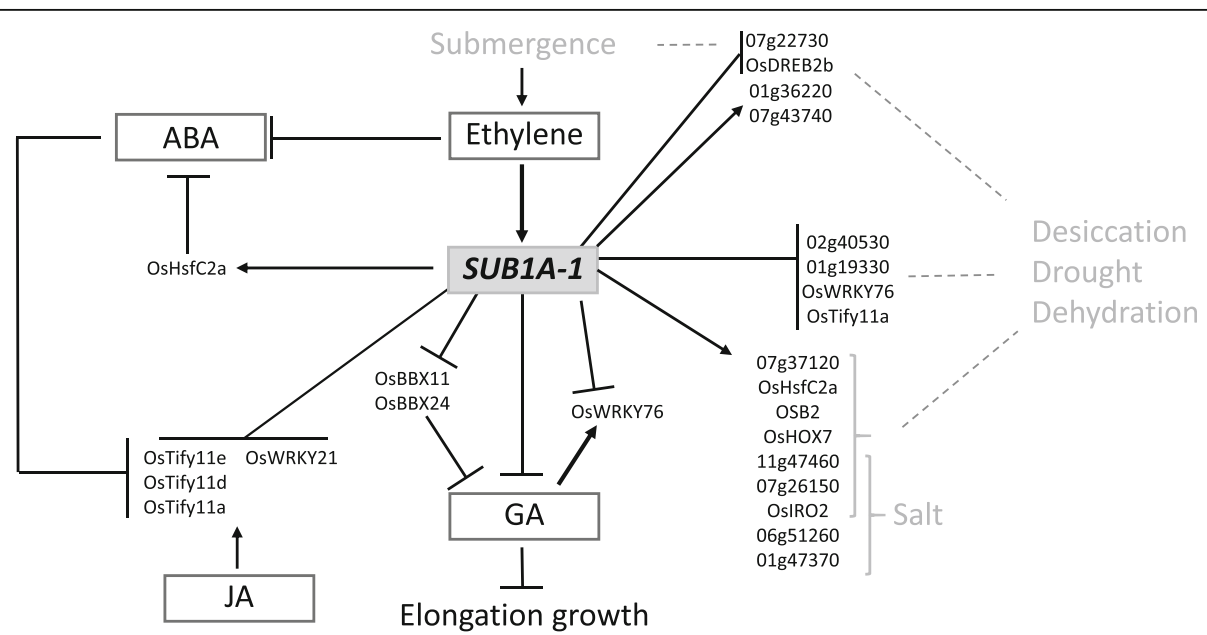

Fig. 3 Differentially expressed transcription factor genes in IR64-Sub1. An overview of the transcription factor genes and their related pathways is provided for all genes that are specifically submergence-responsive in IR64-Sub1 which carries the SUB1A-1 allele. The figure is based on a literature review, see text for details 


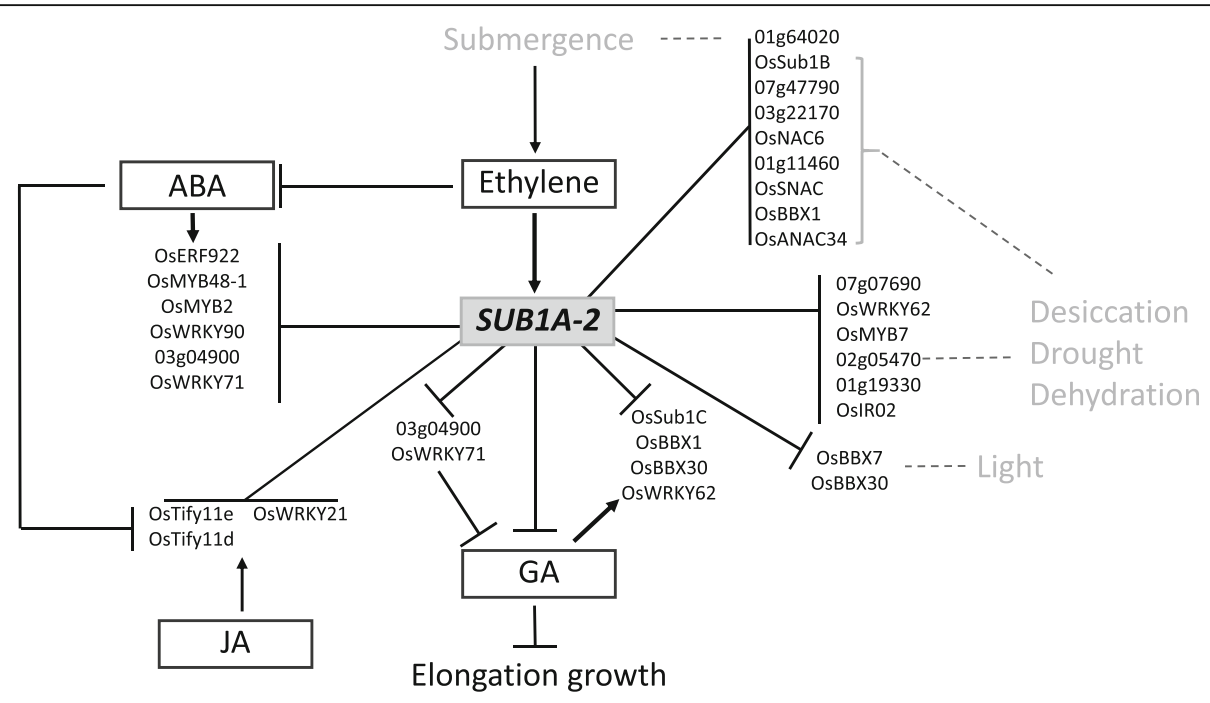

Fig. 4 Differentially expressed transcription factor genes in IR64. An overview of the transcription factor genes and their related pathways is provided for all genes that are specifically submergence-responsive in IR64 which carries the SUB1A-2 allele. The figure is based on a literature review, see text for details

The bHLH OsbHLH016/OSB2 (LOC_Os04g47059) and the HOX OsHOX7 (LOC_Os02g35770) TF genes show upregulation under desiccation stress (Shankar et al., 2016), whereas no information on the bZIP factor (LOC_Os01g36220) is available.

Only two of the above-mentioned genes have previously been reported to be upregulated under submergence and dehydration in IR64-Sub1, namely the bZIP gene (LOC_Os01g36220) and the Zn-finger gene (LOC_Os07g43740) (Fukao et al., 2011). The latter is also upregulated under anoxia, heat and chilling (Ham et al., 2013; Shankar et al., 2016).

\section{Downregulated genes}

In this category, two AP2-type TFs (LOC_Os07g22730, LOC_Os05g27930) have previously been reported as submergence-responsive (Jung et al., 2010). LOC_Os07g22730 belongs to the AP2 group IIIb and was slightly upregulated in M202-Sub1 after $6 \mathrm{~d}$ of submergence but downregulated under anoxia. This gene is also upregulated under desiccation and salt stress (Shankar et al., 2016) and known to be highly expressed in roots and embryos (Rashid, He, Yang, Hussain, and Yan, 2012). The other AP2 factor (LOC_Os05g27930) codes for OSDREB2b, which was reported to be slightly downregulated under submergence in both, M202 and M202Sub1 (Jung et al., 2010). OsDREB2b is a transcriptional activator and is upregulated in the nodes of droughtstressed plants (Todaka et al., 2012). It is also responsive to high and low-temperature stress and over-expressing lines exhibit increased plant survival under drought (Matsukura et al., 2010).
The two downregulated MYB TF genes (LOC_Os02g40530, LOC_01g19330) encode putative calmodulin-binding proteins and are known to play important roles in signal transduction regulating plant development and adaptation responses to different stress conditions (Chantarachot, Buaboocha, Gu, and Chadchawan, 2012). Both genes were reported to be upregulated under drought, desiccation and salt stress whereas downregulated when treated with GA (Chantarachot et al., 2012; Katiyar et al., 2012; Shankar et al., 2016; Xiang et al., 2017).

The other downregulated genes in this category code for OsWRKY76 (LOC_Os09g25060), OsTIFY11a/OsJAZ9 (LOC_Os03g08310) and a RING-H2 finger gene (LOC_Os02g35329). OsWRKY76 is constitutively expressed in vegetative and reproductive tissues and upregulated under desiccation, salinity, auxin, GA and MeJA treatments (Ramamoorthy et al., 2008; Shankar et al., 2016). In contrast, OsTIFY11a/JAZ9 is specifically expressed in young panicles (Ye et al., 2009). OsTIFY11a/JAZ9 is widely stress-responsive and induced by JA, wounding, drought, desiccation, salt and cold stress but downregulated by ABA treatment (Shankar et al., 2016). It was shown that overexpression of TIFY11al $J A Z 9$ enhances shoot growth in rice under salt and mannitol treatment (Ye et al., 2009). OsTIFY11a/JAZ9 interacts with OsCOI1 suggesting its involvement in JA signalling as a transcriptional regulator for salt stress tolerance (Wu, Ye, Yao, Zhang, and Xiong, 2015). No information was found for the RING-H2 finger gene.

\section{Submergence-responsive TFs in IR64}

There were 26 submergence-responsive TF genes specific to IR64, excluding the above-mentioned MYB 
factor LOC_01g19330 common to both genotypes. The genes encode: 5 AP2 domain TFs, 4 NAC, 4 WRKY, 4 MYB, 1 B3 DNA binding protein and a number of different $\mathrm{Zn}$-finger proteins. All of these genes were downregulated under submergence (Fig. 1b; Table 2) and an overview of the data is provided in Fig. 4. Documented studies of these TF genes are summarised below based on their response to submergence, hormone signalling pathways and other abiotic and biotic stresses.

The most responsive gene in IR64 was LOC_Os07g07690, an uncharacterized gene with a putative Jas-domain (pham16135), plant homeodomain (PHD) and Zn-finger domain (cd15539, pfam00628, smart00249). The second most downregulated gene, interestingly, was SUB1B (LOC_Os09g11480), one of the three ERF factors within the Sub1 QTL (Xu et al., 2006) (Table 2, Fig. 2 and Additional file 1: Figure S1a). SUB1B is reportedly not responsive to GA or ethylene (Fukao et al., 2006; Xu et al., 2006). The other AP2 TF genes include SUB1C (LOC_Os09g11460) and three additional ERFs (LOC_Os03g22170, LOC_Os07g47790 and OsERF922: LOC_Os01g54890). The SUB1C gene was reported to be induced by GA and ethylene and upregulated under submergence in the intolerant japonica variety M202, which naturally lacks the SUB1A gene (Fukao et al., 2006). The same study also suggested that $S U B 1 A-1$ negatively regulates $S U B 1 C$ expression. Our results are in agreement with that finding and suggest that $S U B 1 A-2$ also negatively regulates SUB1C under submergence (Fig. 2). However, downregulation of SUB1C was not observed in IR64-Sub1 suggesting an alternate regulatory pathway. One of the recent studies revealed that desiccation stress could also downregulate SUB1C and SUB1B in IR64 plants (Shankar et al., 2016). The three other AP2/ERF genes have also been reported from studies on submergence tolerance. OsERF067 (LOC_Os07g47790) was shown to be upregulated in M202 under submergence and in IR64 under dehydration. This gene was seen to be upregulated by SUB1A during submergence and dehydration in M202Sub1 and IR64-Sub1 suggesting a downstream position in the SUB1A pathway (Fukao et al., 2011). Contrastingly, another study reported downregulation of OsERF067 in M202 under submergence but upregulation in M202Sub1 (Jung et al., 2010). The same study showed a similar expression pattern for OsERF066 (LOC_Os03g22170). Both of these AP2 factors are downregulated under GA treatment (Xiang et al., 2017), involved in cytokinin response (Lara et al., 2004) and are positively responsive to anaerobic germination in Nipponbare (Magneschi and Perata, 2009). The gene OsERF922 was shown to be upregulated in M202-Sub1 after 6 days of submergence but downregulated in M202 after 1 day of submergence (Jung et al., 2010). OsERF922 is strongly induced by ABA, salt treatment, and biotic stress. A study on the crosstalk between abiotic and abiotic stress signalling through modulation of ABA levels further suggests that OsERF922 functions as a transcriptional activator (Liu, Chen, Liu, Ye, and Guo, 2012).

Of the 4 NAC genes downregulated in IR64, two have been reported to respond to various biotic and abiotic stresses, including submergence. OsANAC34 (LOC_Os03g04070) is upregulated under submergence, desiccation and by rice stripe virus infection but downregulated under GA (Nuruzzaman et al., 2010; Shankar et al., 2016; Xiang et al., 2017). The OsNAC6/SNAC2 (LOC_Os01g66120) gene contains ABRE and CBE elements in its promoter and is upregulated under drought, cold and submergence stress (Nuruzzaman et al., 2010). These two genes are responsive in Nipponbare, suggesting they are not under the control of $S U B 1 A$, which is absent in this rice variety. OsNAC6/SNAC2 was also reported to be upregulated under desiccation and salinity stress in a drought-tolerant rice (N22) mutant (Shankar et al., 2016).

The C3HC4 Zn finger (LOC_Os01g11460) and B-Box $\mathrm{Zn}$ finger OsBBX1 (LOC_Os01g10580) TF genes were upregulated under submergence in the intolerant variety M202, the former also in M202-Sub1, and in IR64 upon dehydration (Fukao et al., 2011). OsBBX1 is highly expressed in seeds, roots and the endosperm, and is induced by auxin, GA and cytokinin (Huang et al., 2012). The bZIP TF factor LOC_Os01g64020 was found to be downregulated under submergence in IR64 plants in our study and was reported to be nitrogen-responsive in studies in rice and Arabidopsis (Obertello, Shrivastava, Katari, and Coruzzi, 2015).

The induction of ethylene in response to submergence is known to contribute to a decline in ABA and an increase in GA thereby regulating elongation growth (see above). ABA in-turn is involved in the regulation of the crosstalk between JA and SA signalling pathways (Liu et al., 2012). A complex interplay and fine-tuning of hormonal responses is therefore likely to determine the level of submergence tolerance.

Several studies have shown that four of the IR64downregulated TFs are upregulated $(\uparrow)$ or downregulated $(\downarrow)$ in response to ABA: OsMYB48-1 (LOC_Os01g74410)

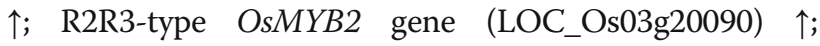
OsWRKY90 (LOC_Os09g30400) $\uparrow$ and OsERF066 (LOC_Os03g22170) $\uparrow$. These genes were also reported to be responsive to PEG-induced water stress (OsMYB48-1 $\uparrow \downarrow), \mathrm{H}_{2} \mathrm{O}_{2}$ (OsMYB48-1 $\left.\uparrow\right)$, drought (OsMYB48-1 $\uparrow$,

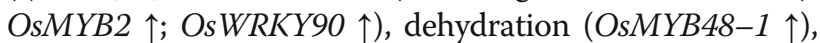
desiccation (OsMYB2 $\uparrow)$, salinity (OsMYB48-1 $\uparrow$, OsMYB2 $\uparrow)$ and cold (OsMYB48-1 $\uparrow$, OsMYB2 $\uparrow)$. Other stress responses include pathogen attack (OsWRKY90 $)$, wound-

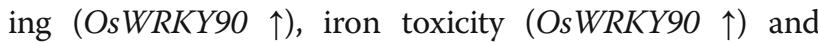
senescence (OsWRKY90 $\uparrow$ ) (Ricachenevsky, Sperotto, Menguer, and Fett, 2010) (Katiyar et al., 2012; Xiong et al., 
2014). OsMYB48-1 also plays a positive role in drought and salinity tolerance by regulating ABA synthesis (Xiong et al., 2014).

The other important set of genes is GA-responsive, including OsSUB1C ( $\uparrow)$ and the $\mathrm{Zn}$ finger OsBBX30 (LOC_Os12g10660) mostly expressed in vegetative tissues and mature panicles $(\uparrow)$, the B-Box factors OsBBX1 and OsBBX7 ( $\uparrow$ ), the WRKY gene OsWRKY62 (LOC_Os09g25070), highly expressed in mature leaves and young roots ( $\uparrow$ ), OsWRKY71/OsEXB1 (LOC_Os02g08440) $(\downarrow)$, OsANAC34 $(\downarrow)$ and two MYB TF genes (OsMYB2 and LOC_Os03g04900) ( $\downarrow)$. Expression of these TFs was also responsive to light (OsBBX30 $\uparrow)$, auxin (OsBBX30 $\uparrow, O s B B X 1$

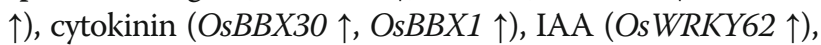

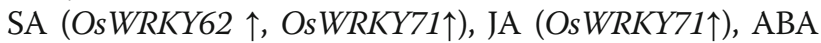

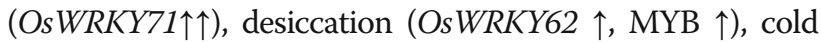

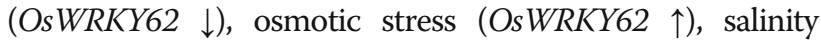
(MYB $\uparrow$ ), and pathogen attack (OsWRKY62 $\uparrow$ ) (Ramamoorthy et al., 2008; Shankar et al., 2016; Xiang et al., 2017). OsBBX7 (LOC_Os02g49230) is highly expressed in leaves, hull and endosperm and is light-responsive. It displays a diurnal expression pattern, i.e. upregulated at night under short-day conditions and high expression during light compared to dark under long-day conditions (Huang et al., 2012). OsWRKY62 was also observed to be nonresponsive to $\mathrm{ABA}$ treatment and therefore likely acts in an ABA-independent signalling pathway. OsWRKY71 is a general stress-responsive gene and was reported to be upregulated by submergence in M202 and dehydration in IR64. However, this response was not observed in M202-Sub1 and IR64-Sub1 (Fig. 2) (Fukao et al., 2011). This gene is also involved in desiccation and salt stress responses in the rice variety N22 (Shankar et al., 2016) and overexpression of OsWRKY71 in rice enhances tolerance to cold (Kim et al., 2016) and resistance to Xanthomonas (Liu, Bai, Wang, and Chu, 2007; Berri et al., 2009; Chujo et al., 2013). The MYB TF gene shares high sequence similarity with MULTIPASS (OsMPS), which regulates plant growth via expansins and was shown to be downregulated by GA and auxin but upregulated by $\mathrm{ABA}$ and cytokinin (Schmidt et al., 2013).

The other TF genes have not been reported to be ABA- or GA-responsive and/or responsive to submergence. OsBBI1 (LOC_Os06g03580) and OsONAC7 (LOC_Os11g05614) have been associated with biotic stress response. OsBBI1 encodes a RING finger protein with E3 ligase activity, which modifies cell wall defence and confers broad-spectrum disease resistance to blast fungus (Magnaporthe oryzae). Expression of OsBBI1 is also induced by the rice blast fungus, benzothiadiazole and SA (Li et al., 2011). OsONAC7 is expressed and highly upregulated in rice stripe virus (RSV) and tungro virus (RTSV) infected plants (Nuruzzaman et al., 2010). The NAC TF gene OsSNAC (LOC_Os07g37920) was shown to be downregulated under drought stress in the intolerant variety Nipponbare (Nuruzzaman et al., 2010) but upregulated under desiccation stress in the rainfed varieties N22 and Pokkali (Shankar et al., 2016).

Limited information is available for the remaining genes downregulated in IR64. An in-silico study proposed B3 domain factor LOC_Os08g06120 to colocalize with a QTL for seed width (Peng and Weselake, 2013). The PHD Zn finger gene OsMYB7 (LOC_Os08g43550) and the CCT motif orphan gene (LOC_Os02g05470) have no defined function but were both downregulated under desiccation stress in IR64 (Shankar et al., 2016). OsWRKY19 (LOC_Os05g49620) was shown to be constitutively low expressed across different tissues and was upregulated by IAA and SA with no clear involvement in any stress response (Ramamoorthy et al., 2008).

\section{SUB1A promoter analysis}

A comparative sequence analysis of the promoter region of the SUB1A-1 and SUB1A-2 alleles (Additional file 2: Table S5) revealed the presence of 13 SNPs. Out of these, 5 SNPs gave rise to allele-specific putative cisregulatory elements that could be targets of upstream TFs. (Fig. 5; Additional file 3: Figure S2).

In the SUB1A-1 allele, SNP4, SNP5, and SNP13 create putative motifs (Fig. 5). SNP4 constitutes a Site I (SITEIOSPCNA) motif that resembles the G-box and was discovered in the rice PROLIFERATING CELL NUCLEAR ANTIGEN (PCNA) promoter. Promoter deletion studies had shown that the Site I element is a transcriptional activation site of PCNA and that it is essential for meristematic tissue-specific expression (Kosugi, Suzuka, and Ohashi, 1995). SNP5 resulted in two distinct cis-elements in the two SUB1A alleles (see below) creating a CAREOSREP1 motif in SUB1A-1. This motif was shown to confer GA-mediated increased expression of a proteinase gene in rice seeds (Sutoh and Yamauchi, 2003). SNP13 creates the other constituent of the Site I bipartite motif, i.e., the Site II element (SITEIIATCYTC). This motif was also formed by SNP9 in the SUB1A-2 allele (see below). In addition to $P C N A$, Site II elements are present in the promoters of many Arabidopsis cell cycle, mitochondrial, ribosomal and cytochrome genes (Tremousaygue et al., 2003; Welchen and Gonzalez, 2005). They are also overrepresented in the promoters of genes encoding nuclear components of the oxidative phosphorylation complex in Arabidopsis and rice (Welchen and Gonzalez, 2005; Welchen and Gonzalez, 2006). Promoter::GUS studies showed that the Site II motif is sufficient to mediate gene expression in shoot and root meristems, as well as in anthers (Kosugi and Ohashi, 1997). It is recognised by TCP and bHLH TFs, which play crucial roles in hormone response pathways, light and abiotic stress responses (Danisman, 2016). Importantly, the position of the Site II element specific to the SUB1A-1 


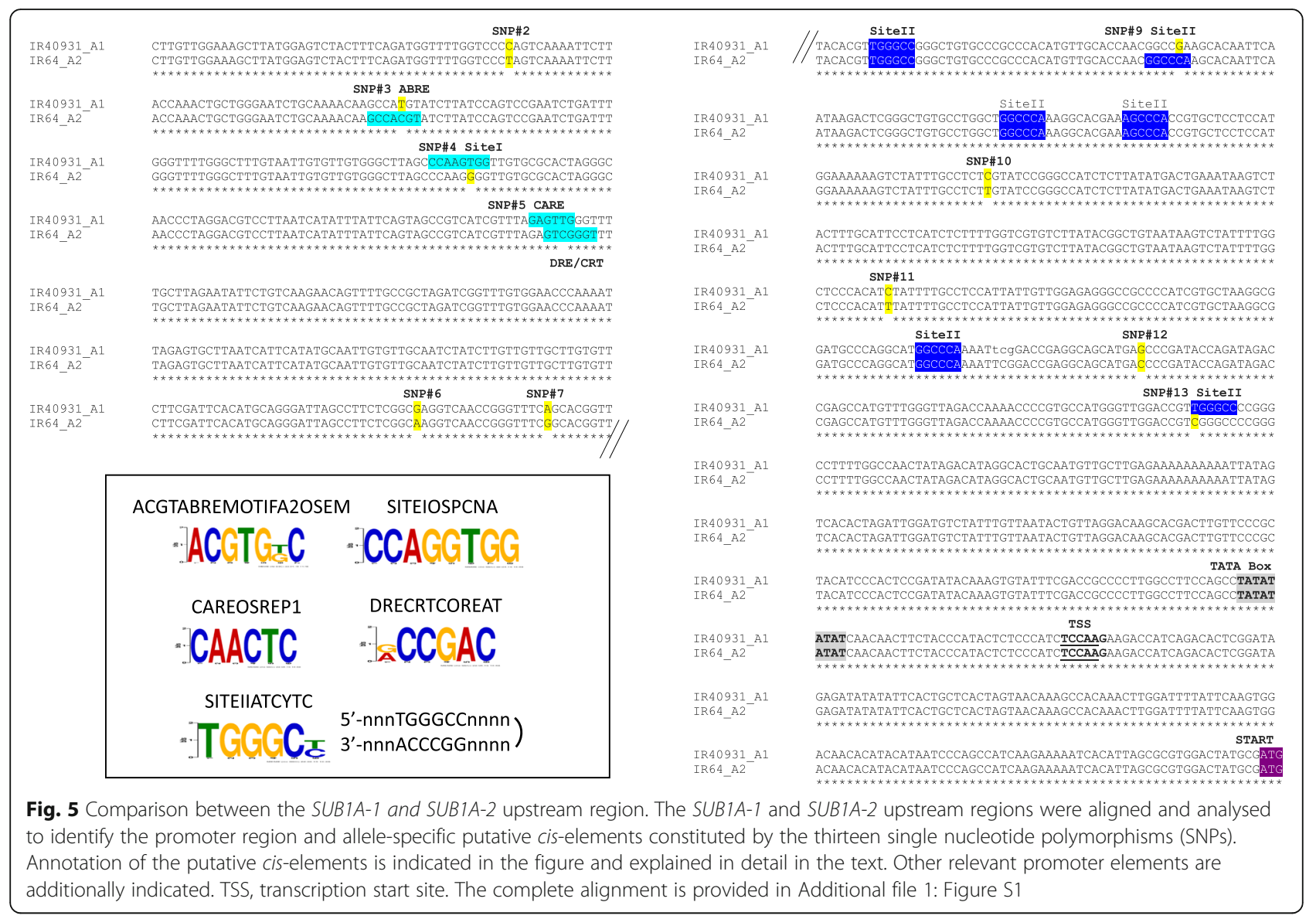

allele is closer to the transcription start site (TSS), which was shown to be the more active position, thus makes this element more relevant in SUB1A-1 allele compared to SUB1A-2, where it is located further upstream (Tremousaygue et al., 2003; Welchen and Gonzalez, 2006).

The Sub1A-2 allele had three SNPs (SNP3, SNP5, SNP9) creating allele-specific putative cis-regulatory elements. SNP3 generates an ABRE motif (ACGTABREMOTIFA2OSEM) in the negative strand, a motif present in many ABA-responsive genes (Hattori, Totsuka, Hobo, Kagaya, and Yamamoto-Toyoda, 2002; Narusaka et al., 2003). SNP5 constitutes the core of the DRE/CRT (dehydration-responsive element/C-repeat; DRECRTCOREAT) motif present in many drought-responsive Arabidopsis and rice genes (Dubouzet et al., 2003; Qin et al., 2004; Diaz-Martin, Almoguera, Prieto-Dapena, Espinosa, and Jordano, 2005; Skinner et al., 2005; Suzuki, Ketterling, and McCarty, 2005). As is the case for SNP13 in the SUB1A-1 allele, SNP9 gave rise to a Site II element (SITEIIATCYTC) but was located further upstream of the TSS as mentioned above.

A third Site II element is present in both alleles (Fig. 5). In addition to Site I and Site II elements, a telo-box (5'AAACCCTAA-3') was identified in the PCNA promoters and shown to act as a transcriptional activator (Tremousaygue et al., 2003). In the SUB1A alleles, no perfect match for the telo-box could be found, however, two truncated telo-box motifs (5'-ACCCTA-3' and 5'-AAACCCT$\left.3^{\prime}\right)$ are present near the $S U B 1 A-2$ and $S U B 1 A-1$ specific Site II elements, respectively (Fig. 5).

\section{Promoter analysis of the DEGs}

The analysis of the $2 \mathrm{~Kb}$ upstream regions of the DEGs identified 32 distinct motifs present in at least one of the genes (Table 3). These motifs can be categorized into four groups based on their relatedness to hypoxia \& light, energy \& sugar metabolism, transcription \& cell division, and hormones \& stress.

Under submergence, plants are exposed to hypoxia and low light conditions and in agreement with this, four anaerobic consensus motifs (ANAERO1-4) and the light response motif GT1CONC4:C36SENSUS were found in most DEGs. The anaerobic consensus motifs were previously identified in genes involved in the anaerobic fermentative pathway (Mohanty, Krishnan, Swarup, and Bajic, 2005). The GT1 motif has been found in light-regulated genes and is known to stabilize the TATA box complex (Terzaghi and Cashmore, 1995; Villain, 
Table 3 Putative cis-elements identified in differentially expressed TF genes

\begin{tabular}{|c|c|c|c|c|}
\hline CATERGORY & MOTIF & IR64 down (\#27 genes) & IR64-Sub1 down (\#7 genes) & IR64-Sub1 up (\#13 genes) \\
\hline \multirow[t]{5}{*}{ Hypoxia \& light } & GT1CONC4:C36SENSUS & 26 & 7 & 13 \\
\hline & ANAERO2CONSENSUS & 18 & 6 & 8 \\
\hline & ANAERO1CONSENSUS & 16 & 5 & 8 \\
\hline & ANAERO3CONSENSUS & 9 & 2 & 4 \\
\hline & ANAERO4CONSENSUS & 2 & 0 & 1 \\
\hline \multirow[t]{16}{*}{ Energy } & PYRIMIDINEBOXOSRAMY1A & 24 & 7 & 10 \\
\hline & TATCCAOSAMY & 17 & 4 & 12 \\
\hline & CGACGOSAMY3 & 16 & 7 & 8 \\
\hline & TATCCAYMOTIFOSRAMY3D & 9 & 1 & 8 \\
\hline & CAREOSREP1 & 23 & 5 & 7 \\
\hline & SITEIIATCYTC & 21 & 5 & 10 \\
\hline & SITEIIBOSPCNA & 1 & 0 & 0 \\
\hline & SITEIIAOSPCNA & 0 & 0 & 1 \\
\hline & SITEIOSPCNA & 2 & 0 & 2 \\
\hline & AACACOREOSGLUB1 & 13 & 3 & 3 \\
\hline & PROLAMINBOXOSGLUB1 & 5 & 1 & 5 \\
\hline & GCN4OSGLUB1 & 8 & 1 & 0 \\
\hline & ACGTOSGLUB1 & 4 & 2 & 3 \\
\hline & GARE1OSREP1 & 4 & 4 & 1 \\
\hline & GARE2OSREP1 & 0 & 3 & 2 \\
\hline & BP5OSWX & 3 & 2 & 2 \\
\hline \multirow[t]{7}{*}{ Transcription \& cell devision } & BIHD1OS & 25 & 7 & 12 \\
\hline & TATABOXOSPAL & 16 & 4 & 7 \\
\hline & TATABOX1 & 2 & 1 & 0 \\
\hline & E2FCONSENSUS & 13 & 1 & 10 \\
\hline & E2F1OSPCNA & 2 & 0 & 0 \\
\hline & LEAFYATAG & 3 & 0 & 4 \\
\hline & WUSATAg & 6 & 0 & 1 \\
\hline \multirow[t]{4}{*}{ Hormones and stress } & DRECRTCOREAT & 17 & 6 & 7 \\
\hline & ACGTABREMOTIFA2OSEM & 9 & 4 & 5 \\
\hline & ABREOSRAB21 & 6 & 4 & 2 \\
\hline & IRO2OS & 3 & 1 & 0 \\
\hline
\end{tabular}

Mache, and Zhou, 1996; Buchel, Brederode, Bol, and Linthorst, 1999; Le Gourrierec, Li, and Zhou, 1999; Zhou, 1999).

Sixteen of the identified motifs were related to energy and sugar metabolism, and studies associated with some of these motifs are detailed below. RAMY motifs, identified in alpha-amylase genes, were highly represented in all four groups of DEGs (up and downregulated DEG in IR64 and IR64-Sub1). The RAMY3D motif (TATCCAY) was found in the rice $R A m y 3 D$ alpha-amylase gene promoter and is responsible for sugar repression (Toyofuku, T-a, and Yamaguchi, 1998). The AMY3 motif (CGACGO) is present in the GC-rich regions of amylase genes and functions as a coupling element for the G-box element (Hwang, Karrer, Thomas, Chen, and Rodriguez, 1998). The other AMY motif (TATCCA) is a binding site for OsMYBS1, OsMYBS2 and OsMYBS3 TFs, which mediate sugar and hormone regulation of alpha-amylase gene expression (Lu, Lim, and Yu, 1998; Lu, Ho, Ho, and Yu, 2002; Chen, Chiang, Tseng, and Yu, 2006). GARE and CARE motifs are necessary for GA regulation of alphaamylases genes (Sutoh and Yamauchi, 2003) and the BP5OSWX motif acts as a transcriptional activator in the waxy gene (Zhu, Cai, Wang, and Hong, 2003). The GARE motif was present in about $50 \%$ of the IR64-Sub1 upregulated genes and less frequent (15\%) in the downregulated 
genes in both genotypes (Table 3). The GARE and RAMY1A (PYRIMIDINEBOX) motifs participate in sugar repression and are present in the promoter of the rice alpha-amylase gene RAmy $1 A$ and the barley alphaamylase gene $A m y 2 / 32 b$, both of which are GA inducible (Morita et al., 1998; Mena, Cejudo, Isabel-Lamoneda, and Carbonero, 2002). The four identified GLUB1 motifs have been associated with the rice glutelin gene OsGluB-1. They are related to energy metabolism and are proposed to control endosperm-specific expression (Washida et al., 1999; Wu, Washida, Onodera, Harada, and Takaiwa, 2000; Qu, Xing, Liu, Xu, and Song, 2008). Glutelins are primary energy storage proteins and the relevance of these motifs remains to be assessed with respect to submergence response. Site II motifs as detailed above, were also found in most DEGs.

Seven of the identified motifs have been related to transcription (E2F, EF1, and TATA Box), meristems (LEAFYATAG and WUSATAg) and homeotic development (BIHD1OS). The E2F binding site was present in $80 \%$ of the IR64-Sub1 upregulated genes and less frequent in the downregulated genes (IR64-50\% and IR64-Sub1-10\%). E2F TFs are regulators of the cell cycle and can act as transcriptional activators or repressors (Vandepoele et al., 2005). The meristem motifs were rare compared to BIHD1OS, which was present in most DEGs. The BIHD1OS motif has been reported to be the binding site for OsBIHD1, a rice BELL homeodomain TF that integrates ethylene and BR hormonal responses (Liu et al., 2017).

Four of the DEG motifs were associated with hormone and stress responses. The drought-related DRE/CRT and ABA-responsive ABRE motifs were most frequent (Dubouzet et al., 2003; Qin et al., 2004; Diaz-Martin et al., 2005; Skinner et al., 2005; Suzuki et al., 2005), whereas the Fe starvation related motif IRO2 (Ogo et al., 2006) was present in only about $10 \%$ of the genes. Amongst the groups of DEGs, DRE/CRT and ABRE motifs were most common in the IR64-Sub1 downregulated genes (60-80\%) compared to the other two groups (Table 3).

\section{Discussion}

All SUB1A-related studies so far have been conducted using contrasting genotypes with and without the SUB1A gene and, therefore, limited information is available on the differential effect of the two alleles. As a first step, the objective of this study was therefore to assess if different sets of TF genes are regulated by the strong SUB1A-1 allele, as present in IR64-Sub1, and the weaker SUB1A-2 allele, naturally present in the wild-type IR64. An earlier study on a range of rice genotypes had shown that both alleles are highly expressed in nodes of submerged plants, however, differences in the expression level in internodes were most pronounced and correlated best with submergence tolerance (Singh et al.,
2010). Therefore, internode tissue was used for this comparative study.

The qPCR analysis of the $2487 \mathrm{TF}$ genes revealed a relatively small number (46) of TF genes that were significantly differentially expressed under submergence. Interestingly, the predominant transcriptional response was reduced expression and only thirteen genes, all in IR64-Sub1, were upregulated under submergence. This, per se, might at least partly explain the higher tolerance level of IR64-Sub1. Further to this, with the exception of an uncharacterized MYB factor, distinct sets of TFs were identified in IR64 and IR64-Sub1, suggesting that the two genotypes employ different regulatory pathways leading to different levels of tolerance.

\section{Association between submergence responsive DEGs and plant hormones}

Nineteen TF genes were specifically submergenceresponsive in IR64-Sub1, making them candidates to explain the higher tolerance of IR64-Sub1 compared to IR64. The upregulated genes were previously reported in studies on drought, salt and other abiotic stresses, but not with respect to submergence stress (Additional file 2: Table S7). In agreement with our data, the OsbZIP4 and the Zn-finger gene LOC_Os07g43740 were found to be upregulated under anoxia (Ham et al., 2013; Shankar et al., 2016).

Available data on the hormonal response of some of these genes (OsHsfC2a, OsBBX11, OsBBX24, OsWRKY76 and OsTIFY11a) suggest that they are part of the ABA/ GA regulatory pathway (Fig. 4). For instance, one of the six upregulated MYB factors (LOC_Os08g06110) was reported to reduce seed dormancy in a rice mutant through ABA-GA antagonism (Wu et al., 2016). OsHsfC $2 a$ is downregulated by ABA, while, GA causes downregulation of OsBBX11 and OsBBX24 (Huang et al., 2012). Additionally, OsBBX11 is regulated by light, which plays a critical role in the submergence response (Das et al., 2009).

Among the genes downregulated in IR64-Sub1 were two AP2-type TFs (OsERF136, OsDREB2b), both of which are submergence-responsive in M202 and M202Sub1 (Jung et al., 2010). Since the wild-type genotype M202 naturally lacks SUB1A, the two AP2 genes might act in a different pathway, independent of SUB1A. $D R E B 2 b$ has also been reported to function as a transcriptional activator under low- and high-temperature stress and it enhances drought tolerance when overexpressed (Matsukura et al., 2010; Todaka et al., 2012). Similarly, SUB1A has been implicated with enhanced recovery growth after severe drought (Fukao et al., 2011) and submerged plants generally survive longer in cooler water (Das et al., 2009). These similarities between $S U B 1 A$ and DREB2b make the latter an interesting candidate gene for integrating these signal responses. 
In contrast to IR64-Sub1, the DEGs in IR64 were all downregulated (Fig. 5). The most highly downregulated gene encodes an uncharacterized protein, with putative Jas- and PHD Zn-finger domains, suggesting that it may be a part of the JA signalling pathway and/or a regulator of development. The second most highly responsive gene was OsSUB1B, followed by WRKY and additional ERF/AP2 factors, including OsSUB1C. Interestingly, both $O s S U B 1 B$ and OsSUB1C are, despite also in IR64-Sub1, only differentially expressed (downregulated) in IR64. OsSUB1B is non-responsive to GA and ethylene and no tolerant-specific allele has been identified for this gene (Xu et al., 2006). A clear downregulation of OsSUB1B under submergence in IR64 justifies more in-depth analysis of its function and regulation of expression. In contrast, tolerance-specific alleles for OsSUB1C are known and its induction by GA and ethylene specifically in M202-Sub1 has been documented (Fukao et al., 2006; $\mathrm{Xu}$ et al., 2006). These studies also indicated that SUB1A-1 might be a negative regulator of OsSUB1C. Downregulation of OsSUB1C in IR64 suggests that the OsSUB1A-2 allele can likewise act as a negative regulator of OsSUB1C, directly or indirectly.

The three AP2/ERF genes downregulated in IR64 (OsERF067, OsERF066, OsERF922) had previously been shown to be upregulated in M202-Sub1 under submergence (Jung et al., 2010). This was not observed in IR64Sub1 in this study, however, their downregulation in IR64 might at least partly explain a lower level of submergence tolerance. OsERF922 may be of particular interest here since this gene acts as a transcriptional activator and modulator of ABA levels (Liu et al., 2012).

The $B B X 1$ gene had previously been shown to be submergence induced in the variety M202, which lacks the SUB1A gene (Fukao et al., 2011). This is in contrast to the observed downregulation of $B B X 1$ in IR64 in our study, suggesting that $B B X 1$ might be under the control of the SUB1A-2 allele contributing to the higher tolerance level of IR64 compared with M202.

As was the case for IR64-Sub1, a number of the IR64 downregulated DEGs have been associated with GA and ABA regulation. Among the GA-responsive genes, the MYB TF (LOC_Os03g04900) might be of particular interest as it is similar to OsMPS, which acts as a regulator of plant growth by suppressing expansin genes and is downregulated by GA and upregulated by ABA treatment (Schmidt et al., 2013). Suppression of expansins has previously been shown to be a critical component of the $S U B 1 A$-mediated quiescence response and it will be interesting to assess if this MYB factor functions in a way similar to OsMPS.

Genes upregulated by ABA (OsMYB2, OsWRKY90, OsERF922 and OsMYB48-1) activate the ABA synthesis pathway and OsMYB48-1, specifically, has been reported to induce other ABA-responsive genes under drought (Xiong et al., 2014). On the other hand, four genes (OsSUB1C, OsBBX1, OsBBX30, OsWRKY62) have antagonistic effects to $\mathrm{ABA}$ and respond positively to GA (Ramamoorthy et al., 2008; Huang et al., 2012; Shankar et al., 2016). The fact that all of these genes are downregulated in IR64 under submergence suggests that at least part of the GA signalling pathway is inactivated, as required for submergence tolerance. That these genes have not identified as differentially expressed in IR64Sub1 is not necessarily a contradiction but instead suggests that submergence tolerance might be related to low or neutral expression of these genes.

\section{Role of iron uptake and JA in submergence tolerance}

Iron solubility and availability in plants is altered based on soil $\mathrm{pH}$ and submergence conditions and can result in excess iron availability. Difficulties in iron uptake cause chlorosis whereas iron overload or increased uptake leads to oxidative stress and permanent cell and tissue damage (Zheng 2010). One of the four TFs that showed a constitutive difference between the two genotypes, OsIRO2, was constitutively lower expressed in IR64-Sub1 (Fig. 2a). IRO2 has so far only been described in relation to Fe and it was shown to be specifically induced by Fe deficiency (Ogo et al., 2006; Kobayashi et al., 2007). Although the specific role during submergence remains to be determined, the higher expression of the IRO2 in IR64 might suggest a higher Fe uptake in this cultivar resulting in oxidative stress and cell damage.

As expression of IRO2 is not induced by submergence, a specific role for IRO2 is perhaps unlikely, however, it might be possible that the $S U B 1 A-2$ allele specifically acts as an enhancer of OsIRO2 thereby causing a secondary, negative effect under submergence. It will, therefore, be interesting to compare the performance of IR64 and IR64-Sub1 under Fe deficiency conditions and quantify plant iron content.

Five of the other TF genes identified in this study (OsTIFY11e, OsTIFY11d, OsWRKY76, OsTIFY11a, OsWRKY71) were reported to be upregulated by JA and downregulated by ABA (Ye et al., 2009; Ranjan et al., 2015; Yang et al., 2015; Shankar et al., 2016). Additionally, WRKY76 and WRKY71 are also upregulated by GA (Ramamoorthy et al., 2008; Shankar et al., 2016). This is well in support of the finding that fine-tuning of GA and ABA levels under submergence is a major component of regulating elongation growth in rice (Fukao et al., 2006; Fukao and Bailey-Serres, 2008) and studies on Arabidopsis have further shown that JA plays a crucial role in protecting plants during post-submergence reoxygenation (Yuan et al., 2017). The genes identified in this study might therefore play a role in integrating various hormonal signals and analysing their expression in 
IR64 and IR64-Sub1 plants upon de-submergence will provide more insights into the role of $S U B 1 A-2$ during recovery growth.

A significant number of DEGs controlled by submergence have also been described as cold stress responsive. In addition, it has been shown that plant survival in cooler submergence water is higher (Das et al., 2009). Therefore, higher expression of these cold-induced genes in IR64-Sub1 may contribute to its higher tolerance level. For the genes listed in Additional file 2: Table S7, cold responsiveness was also observed in M202 and M202-Sub1 (Jung et al., 2010).

\section{Putative cis-regulatory elements}

The diversity of the putative regulatory cis-elements identified in the promoters of the DEGs (Table 3) is well in agreement with the diverse environmental stimuli associated with submergence requiring plants to adjust to low oxygen, light and temperature, alter their energy and sugar metabolism, cell divisions, as well as hormone-regulated growth. For instance, the light-responsive motif (GT1CONC4:C36SENSUS) occurred in all genes except one, suggesting that low-light is an integral signal that can trigger both, up- and downregulation of genes. Likewise, the majority of genes appears to be responsive to hypoxia, as indicated by the presence of the anaerobic consensus motifs, ANAERO1CONSENSUS and ANAERO2CONSENSUS, as well as the PYRIMIDINEBOXOSRAMY1A, found in alpha-amylase genes. The AMY motif (TATCCA) appears to be more frequent in IR64-Sub1 downregulated genes, whereas the AMY3 motifs (CGACG), which functions as a G-box coupling element, is more prevalent in upregulated genes. The former is the binding site of $O s M Y B s$, which mediate sugar and hormone regulation of alpha-amylase genes (Lu et al., 1998; Lu et al., 2002; Chen et al., 2006). Since maintenance of a higher level of soluble sugars has been implicated with tolerance and better recovery growth (Singh et al., 2001; Das et al., 2005; Fukao et al., 2006) these TF genes might be part of the regulatory pathway controlling sugar consumption under submergence. Despite the importance of ABA in submergence tolerance, surprisingly few genes had ABRE elements. In contrast, the DRE/CRT element was more frequent, which, in conjunction with the identified differential expression of DREB2b (Matsukura et al., 2010; Todaka et al., 2012), reinforces a possible relationship between submergence and drought tolerance (Fukao et al., 2011).

Among the most interesting elements identified in the promoter analysis is the bi-partite Site II element. This motif was frequently present in the DEGs and also distinguished the SUB1A-1 and SUB1A-2 alleles at the SNPs (Fig. 5). Since this motif has been implicated with meristem-specific expression (Tremousaygue et al., 2003; Welchen and Gonzalez, 2006) its presence might indicate that control of cell division, in addition to elongation growth, is important for tolerance. This is supported by promoter::GUS reporter studies that showed specific SUB1A-1 expression in the base of growing leaves (Singh et al., 2010). It will be interesting to study this motif in more detail and to determine if the position closer to the TTS of the SUB1A-1-specific Site II element enhances the capacity of tolerant plants to suppress cell division under submergence. In relation to this, it is relevant that the two SUB1A alleles are also distinct in their coding region for a MAPK3 phosphorylation target site, which is specifically present in SUB1A1 (Singh and Sinha, 2016). The same study also showed that both, SUB1A-1 and SUB1A-2, physically interact with MAPK3 but that the interaction with SUB1A-2 was weaker. Thus, SUB1A-2 would still be a component of this regulatory pathway but might be less efficient. It will indeed be interesting to investigate the role of SUB1A in regulating cell division in more detail and to establish whether SUB1A-2 binds to the MAPK3 promoter, as was shown for SUB1A-1 (Singh and Sinha, 2016).

\section{Conclusion}

This study identified distinct sets of submergenceresponsive transcription factor genes in IR64 and IR64Sub1 which might at least partly explain differences in the level of tolerance between the two genotypes. These genes should now be assessed for putative additive effects with SUB1A and their potential to enhance tolerance. The promoter analysis identified allele-specific site II elements, which should be studied in more detail to establish if SUB1A directly or indirectly suppresses cell division, in addition to its documented role in suppressing cell elongation.

\section{Additional files}

\footnotetext{
Additional file 1: Figure S1. The submergence tolerance locus Sub1 in rice. The Sub1 locus is located on rice chromosome 9 with a variable number of SUB1 genes. In different rice genotypes, SUB1A is either absent or present as different allele. (a). Introgression of the Sub1 locus into the rice variety IR64 (IR64-Sub1) enhanced survival after complete submergence. (b). The photo shows the IRRI demonstration field plot in the Philippines. Phenotyping for submergence tolerance can also be conducted by submerging plants grown in trays (inlay). The SUB1A-1 and SUB1A-2 alleles are both highly expressed in nodes of submerged plants but SUB1A-1 expression is higher in internodes. (c). Schematic illustration based on (Singh et al., 2010). (PPTX 3066 kb)

Additional file 2: Table S1. List of genes represented in Rice TF Primer Platform (Caldana et al., 2007). Complete list of 2508 TF genes, their primer sequences and their corresponding PCR efficiencies. Genes were presented with version 2.0 and version 5.0 RGAP genome annotations. Table S2. Transcription factor genes with and without annotations in version 5.0 and 7.0 of RGAP Pseudomolecule. Table S3. Expression profile of transcription factor genes in IR64 control and stress-treated plants upon submergence. Recorded $\mathrm{Ct}, \mathrm{R}^{2}$, Efficiency and $\Delta \mathrm{Ct}$ values for all reactions. The list of used reference genes and their distribution on the plates is also given. Table S4. Expression profile of transcription factor
} 
genes in IR64-Sub1 control and stress-treated plants upon submergence. Worksheets record Ct, $\mathrm{R}^{2}$, Efficiency and $\Delta \mathrm{Ct}$ values for all reactions. The list of used reference genes and their distribution on the plates is also given. Table S5. Promoter sequences of SUB1A-1 and SUBTA-2. Table S6. Moderated t-tests results from LIMMA package for identifying differentially expressed genes. $P$-values were recorded and FDR corrections were also performed. Table S7. Details about the list of differentially expressed genes and their putative functions. (DOCX $29 \mathrm{~kb}$ )

Additional file 3; Figure S2. Sequence alignment of the SUBTA-1 and SUB1A-2 upstream promoter regions. $2 \mathrm{~kb}$ upstream of the start site (promoter regions) were analysed for SUB1A-1 and SUB1A-2 alleles and putative cis-regulatory elements were identified. (XLSX $3.76 \mathrm{~kb}$ )

\section{Acknowledgments}

This work was supported by the International Rice Research Institute (IRRI; Philippines), the Max Planck Institute of Molecular Plant Physiology (Potsdam, Germany), and the University of Potsdam. Rothamsted Research is supported by the Biotechnology and Biological Sciences Research Council (BBSRC, UK).

\section{Authors' contributions}

SH designed and supervised the study. BMR has developed and provided access to the GPCR analysis pipeline. TDM and SR performed the experiments, NiS and UB updated and re-analysed the qPCR data. NiS, NaS and $\mathrm{SH}$ wrote the manuscript with inputs from UB and BMR. All authors read and approved the final manuscript.

\section{Competing interests}

All authors declare that they have no competing interests.

\section{Publisher's Note}

Springer Nature remains neutral with regard to jurisdictional claims in published maps and institutional affiliations.

\section{Author details}

${ }^{1}$ School of Agriculture, Food and Wine, University of Adelaide, Waite Campus, Plant Genomics Centre, Hartley Grove, Urrbrae, Adelaide, South Australia 5064, Australia. ${ }^{2}$ International Rice Research Institute (IRRI), Los Banos, Philippines. ${ }^{3}$ Intrexon Corp, California, USA. ${ }^{4}$ Section of Plant Breeding and Genetics, School of Integrative Plant Science, Cornell University, Ithaca, NY 14853, USA. ${ }^{5}$ University of Potsdam, Potsdam, Germany. ${ }^{6}$ Rothamsted Research, Plant Science Department, Hertfordshire, Harpenden AL5 2JQ, UK.

Received: 29 September 2017 Accepted: 12 December 2017

\section{Published online: 08 January 2018}

\section{References}

Bailey-Serres J, Fukao T, Ronald P, Ismail A, Heuer S, Mackill D (2010) Submergence tolerant Rice: SUB1's journey from landrace to modern cultivar. Rice (N Y) 3(2-3):138-147

Bailey-Serres J, Voesenek LA (2008) Flooding stress: acclimations and genetic diversity. Annu Rev Plant Biol 59:313-339

Bailey-Serres J, Voesenek LACJ (2010) Life in the balance: a signaling network controlling survival of flooding. Curr Opin Plant Biol 13(5):489-494

Baxter CEL, Costa MMR, Coen ES. 2007. Diversification and co-option of RAD-like genes in the evolution of floral asymmetry. Plant Journal 52(1): 105-113.

Benjamini Y, Hochberg Y (1995) Controlling the false discovery rate - a practical and powerful approach to multiple testing. J Royal Stat Soc Ser BMethodological 57(1):289-300

Berri S, Abbruscato P, Faivre-Rampant O, Brasileiro AC, Fumasoni I, Satoh K, Kikuchi S, Mizzi L, Morandini P, Pe ME, Piffanelli P (2009) Characterization of WRKY co-regulatory networks in rice and Arabidopsis. BMC Plant Biol 9:120

Boyden GS, Donoghue MJ, Howarth DG. 2012. Duplications and Expression of Radialis-Like Genes in Dipsacales. International Journal of Plant Sciences 173(9): 971-983.

Buchel AS, Brederode FT, Bol JF, Linthorst HJM (1999) Mutation of GT-1 binding sites in the pr-1A promoter influences the level of inducible gene expression in vivo. Plant Mol Biol 40(3):387-396
Caldana C, Scheible WR, Mueller-Roeber B, Ruzicic S (2007) A quantitative RT-PCR platform for high-throughput expression profiling of 2500 rice transcription factors. Plant Methods 3:7

Cantrell RP, Reeves TG (2002) The rice genome. The cereal of the world's poor takes center stage. Science 296(5565):53

Chantarachot T, Buaboocha H, Gu H, Chadchawan S (2012) Putative Calmodulinbinding R2R3-MYB transcription factors in Rice (Oryza Sativa L.) Thai J Botany 4(Special Issue):101-112

Chauhan H, Khurana N, Agarwal P, Khurana P (2011) Heat shock factors in rice (Oryza Sativa L.): genome-wide expression analysis during reproductive development and abiotic stress. Mol Gen Genomics 286(2):171-187

Chen PW, Chiang CM, Tseng TH, Yu SM (2006) Interaction between rice MYBGA and the gibberellin response element controls tissue-specific sugar sensitivity of alpha-amylase genes. Plant Cell 18(9):2326-2340

Chow CN, Zheng HQ, Wu NY, Chien CH, Huang HD, Lee TY, Chiang-Hsieh YF, Hou PF, Yang TY, Chang WC (2016) PlantPAN 2.0: an update of plant promoter analysis navigator for reconstructing transcriptional regulatory networks in plants. Nucleic Acids Res 44(D1):D1154-D1160

Chujo T, Miyamoto K, Shimogawa T, Shimizu T, Otake Y, Yokotani N, Nishizawa Y, Shibuya N, Nojiri H, Yamane H, Minami E, Okada K (2013) OsWRKY28, a PAMP-responsive transrepressor, negatively regulates innate immune responses in rice against rice blast fungus. Plant Mol Biol 82(1-2):23-37

Danisman S (2016) TCP transcription factors at the Interface between environmental challenges and the Plant's growth responses. Front Plant Sci 7

Das KK, Sarkar RK, Ismail AM (2005) Elongation ability and non-structural carbohydrate levels in relation to submergence tolerance in rice. Plant Sci 168(1):131-136

Das KK, Panda D, Sarkar RK, Reddy JN, Ismail AM (2009) Submergence tolerance in relation to variable floodwater conditions in rice. Environmental and Experimental Botany 66(3): 425-434.

Diaz-Martin J, Almoguera CN, Prieto-Dapena P, Espinosa JM, Jordano J (2005) Functional interaction between two transcription factors involved in the developmental regulation of a small heat stress protein gene promoter. Plant Physiol 139(3):1483-1494

Dubouzet JG, Sakuma Y, Ito Y, Kasuga M, Dubouzet EG, Miura S, Seki M, Shinozaki K, Yamaguchi-Shinozaki K (2003) OsDREB genes in rice, Oryza Sativa L., encode transcription activators that function in drought-, high-salt- and coldresponsive gene expression. Plant J 33(4):751-763

Fukao T, Bailey-Serres J (2004) Plant responses to hypoxia-is survival a balancing act? Trends Plant Sci 9(9):449-456

Fukao T, Bailey-Serres J (2008) Submergence tolerance conferred by Sub1A is mediated by SLR1 and SLRL1 restriction of gibberellin responses in rice. Proc Natl Acad Sci U S A 105(43):16814-16819

Fukao T, Xu K, Ronald PC, Bailey-Serres J (2006) A variable cluster of ethylene response factor-like genes regulates metabolic and developmental acclimation responses to submergence in rice. Plant Cell 18(8):2021-2034

Fukao T, Yeung E, Bailey-Serres J (2011) The submergence tolerance regulator SUB1A mediates crosstalk between submergence and drought tolerance in rice. Plant Cell 23(1):412-427

Gao FH, Zhang HL, Wang HG, Gao H, Li ZC (2009) Comparative transcriptional profiling under drought stress between upland and lowland rice (Oryza sativa L.) using CDNA-AFLP. Chinese Science Bulletin 54(19): 3555-3571.

Gibbs J, Greenway H (2003) Mechanisms of anoxia tolerance in plants. I. Growth, survival and anaerobic catabolism. Funct Plant Biol 30(1):1-47

Ham DJ, Moon JC, Hwang SG, Jang CS (2013) Molecular characterization of two small heat shock protein genes in rice: their expression patterns, localizations, networks, and heterogeneous overexpressions. Mol Biol Rep 40(12):67096720

Hattori T, Totsuka M, Hobo T, Kagaya Y, Yamamoto-Toyoda A (2002) Experimentally determined sequence requirement of ACGT-containing abscisic acid response element. Plant Cell Physiol 43(1):136-140

Hattori Y, Nagai K, Furukawa S, Song XJ, Kawano R, Sakakibara H, Wu JZ, Matsumoto T, Yoshimura A, Kitano H, Matsuoka M, Mori H, Ashikari M (2009) The ethylene response factors SNORKEL1 and SNORKEL2 allow rice to adapt to deep water. Nature 460(7258):1026-U1116

Huang J, Zhao X, Weng X, Wang L, Xie W (2012) The rice B-box zinc finger gene family: genomic identification, characterization, expression profiling and diurnal analysis. PLoS One 7(10):e48242

Hwang YS, Karrer EE, Thomas BR, Chen L, Rodriguez RL (1998) Three cis-elements required for rice alpha-amylase Amy3D expression during sugar starvation. Plant Mol Biol 36(3):331-341 
Jung KH, Seo YS, Walia H, Cao PJ, Fukao T, Canlas PE, Amonpant F, Bailey-Serres J, Ronald PC (2010) The submergence tolerance regulator Sub1A mediates stress-responsive expression of AP2/ERF transcription factors. Plant Physiol 152(3):1674-1692

Katiyar A, Smita S, Lenka SK, Rajwanshi R, Chinnusamy V, Bansal KC (2012) Genome-wide classification and expression analysis of MYB transcription factor families in rice and Arabidopsis. BMC Genomics 13:544

Kawahara Y, de la Bastide M, Hamilton JP, Kanamori H, McCombie WR, Ouyang S, Schwartz DC, Tanaka T, Wu JZ, Zhou SG, Childs KL, Davidson RM, Lin HN, Quesada-Ocampo L, Vaillancourt B, Sakai H, Lee SS, Kim J, Numa H, Itoh T, Buell CR, Matsumoto T (2013) Improvement of the Oryza Sativa Nipponbare reference genome using next generation sequence and optical map data. Rice (N Y) 6:4.

Kim CY, Vo KTX, Nguyen CD, Jeong DH, Lee SK, Kumar M, Kim SR, Park SH, Kim JK, Jeon JS (2016) Functional analysis of a cold-responsive rice WRKY gene, OsWRKY71. Plant Biotechnol Rep 10(1):13-23

Kobayashi T, Ogo Y, Itai RN, Nakanishi H, Takahashi M, Mori S, Nishizawa NK (2007) The transcription factor IDEF1 regulates the response to and tolerance of iron deficiency in plants. Proc Natl Acad Sci U S A 104(48):19150-19155

Kosugi S, Ohashi Y (1997) PCF1 and PCF2 specifically bind to cis elements in the rice proliferating cell nuclear antigen gene. Plant Cell 9(9):1607-1619

Kosugi S, Suzuka I, Ohashi Y (1995) 2 of 3 promoter elements identified in a Rice gene for proliferating cell nuclear antigen are essential for Meristematic tissue-specific expression. Plant J 7(6):877-886

Lara MEB, Garcia MCG, Fatima T, Ehness R, Lee TK, Proels R, Tanner W, Roitsch T (2004) Extracellular invertase is an essential component of cytokininmediated delay of senescence. Plant Cell 16(5):1276-1287

Le Gourrierec J, Li YF, Zhou DX (1999) Transcriptional activation by Arabidopsis GT-1 may be through interaction with TFIIA-TBP-TATA complex. Plant J 18(6):663-668

Li W, Zhong SH, Li GJ, Li Q, Mao BZ, Deng YW, Zhang HJ, Zeng LJ, Song FM, He ZH (2011) Rice RING protein OsBBI1 with E3 ligase activity confers broadspectrum resistance against Magnaporthe oryzae by modifying the cell wall defence. Cell Res 21(5):835-848

Liu D, Chen X, Liu J, Ye J, Guo Z (2012) The rice ERF transcription factor OsERF922 negatively regulates resistance to Magnaporthe oryzae and salt tolerance. J Exp Bot 63(10):3899-3911

Liu H, Dong SY, Gu FW, Liu W, Yang GL, Huang M, Xiao WM, Liu YZ, Guo T, Wang H, Chen ZQ, Wang JF (2017) NBS-LRR protein Pik-H4 interacts with OsBIHD1 to balance Rice blast resistance and growth by coordinating ethyleneBrassinosteroid pathway. Front Plant Sci 8

Liu XQ, Bai XQ, Wang XJ, Chu CC (2007) OsWRKY71, a rice transcription factor, is involved in rice defense response. J Plant Physiol 164(8):969-979

Lu CA, Ho THD, Ho SL, Yu SM (2002) Three novel MYB proteins with one DNA binding repeat mediate sugar and hormone regulation of alpha-amylase gene expression. Plant Cell 14(8):1963-1980

Lu CA, Lim EK, Yu SM (1998) Sugar response sequence in the promoter of a rice alpha-amylase gene serves as a transcriptional enhancer. J Biol Chem 273(17):10120-10131

Mackill DJ, Ismail AM, Singh US, Labios RV, Paris TR (2012) Development and rapid adoption of submergence-tolerant (Sub1) Rice varieties. Adv Agron 115(115):299-352

Magneschi L, Perata P (2009) Rice germination and seedling growth in the absence of oxygen. Ann Bot 103(2):181-196

Matsukura S, Mizoi J, Yoshida T, Todaka D, Ito Y, Maruyama K, Shinozaki K, Yamaguchi-Shinozaki K (2010) Comprehensive analysis of rice DREB2-type genes that encode transcription factors involved in the expression of abiotic stress-responsive genes. Mol Gen Genomics 283(2):185-196

Mena M, Cejudo FJ, Isabel-Lamoneda I, Carbonero P (2002) A role for the DOF transcription factor BPBF in the regulation of gibberellin-responsive genes in barley aleurone. Plant Physiol 130(1):111-119

Mittal D, Chakrabarti S, Sarkar A, Singh A, Grover A (2009) Heat shock factor gene family in rice: Genomic organization and transcript expression profiling in response to high temperature, low temperature and oxidative stresses. Plant Physiology and Biochemistry 47(9): 785-795.

Mittal D, Madhyastha DA, Grover A (2012) Gene expression analysis in response to low and high temperature and oxidative stresses in rice: Combination of stresses evokes different transcriptional changes as against stresses applied individually. Plant Science 197: 102-113.

Mohanty B, Krishnan SPT, Swarup S, Bajic VB (2005) Detection and preliminary analysis of motifs in promoters of anaerobically induced genes of different plant species. Ann Bot 96(4):669-681
Morita A, T-a U, Kuroyanagi M, Futsuhara Y, Perata P, Yamaguchi J (1998) Functional dissection of a sugar-repressed a-amylase gene (RAmy1A) promoter in rice embryos. FEBS Lett 423(1):81-85

Nagai K, Hattori Y, Ashikari M (2010) Stunt or elongate? Two opposite strategies by which rice adapts to floods. J Plant Res 123(3):303-309

Narusaka Y, Nakashima K, Shinwari ZK, Sakuma Y, Furihata T, Abe H, Narusaka M, Shinozaki K, Yamaguchi-Shinozaki K (2003) Interaction between two cisacting elements, $\mathrm{ABRE}$ and $\mathrm{DRE}$, in ABA-dependent expression of Arabidopsis rd29A gene in response to dehydration and high-salinity stresses. Plant J 34(2):137-148

Nuruzzaman M, Manimekalai R, Sharoni AM, Satoh K, Kondoh H, Ooka H, Kikuchi S (2010) Genome-wide analysis of NAC transcription factor family in rice. Gene 465(1-2):30-44

Obertello M, Shrivastava S, Katari MS, Coruzzi GM (2015) Cross-species network analysis uncovers conserved nitrogen-regulated network modules in Rice. Plant Physiol 168(4):1830-1843

Ogo Y, Itai RN, Nakanishi H, Inoue H, Kobayashi T, Suzuki M, Takahashi M, Mori S, Nishizawa NK (2006) Isolation and characterization of IRO2, a novel ironregulated bHLH transcription factor in graminaceous plants. J Exp Bot 57(11): 2867-2878

Park SH, Jeong JS, Lee KH, Kim YS, Choi YD, Kim JK (2015) OsbZIP23 and OsbZIP45, members of the rice basic leucine zipper transcription factor family, are involved in drought tolerance. Plant Biotechnology Reports 9(2): 89-96.

Peng FY, Weselake RJ (2013) Genome-wide identification and analysis of the B3 superfamily of transcription factors in Brassicaceae and major crop plants. Theor Appl Genet 126(5):1305-1319

Perez-Rodriguez P, Riano-Pachon DM, Correa LG, Rensing SA, Kersten B, MuellerRoeber B (2010) PInTFDB: updated content and new features of the plant transcription factor database. Nucleic Acids Res 38(Database issue):D822-D827

Qin F, Sakuma Y, Li J, Liu Q, Li YQ, Shinozaki K, Yamagushi-Shinozaki KY (2004) Cloning and functional analysis of a novel DREB1/CBF transcription factor involved in cold-responsive gene expression in Zea Mays L. Plant Cell Physiol 45(8):1042-1052

Qu LQ, Xing YP, Liu WX, Xu XP, Song YR (2008) Expression pattern and activity of six glutelin gene promoters in transgenic rice. J Exp Bot 59(9):2417-2424

Ramamoorthy R, Jiang SY, Kumar N, Venkatesh PN, Ramachandran S (2008) A comprehensive transcriptional profiling of the WRKY gene family in rice under various abiotic and phytohormone treatments. Plant Cell Physiol 49(6): 865-879

Ranjan A, Vadassery J, Patel HK, Pandey A, Palaparthi R, Mithofer A, Sonti RV (2015) Upregulation of jasmonate biosynthesis and jasmonate-responsive genes in rice leaves in response to a bacterial pathogen mimic. Funct Integr Genomics 15(3):363-373

Rashid M, He GY, Yang GX, Hussain J, Yan X (2012) AP2/ERF transcription factor in Rice: genome-wide canvas and Syntenic relationships between monocots and Eudicots. Evol Bioinforma 8:321-355

Riano-Pachon DM, Ruzicic S, Dreyer I, Mueller-Roeber B (2007) PInTFDB: an integrative plant transcription factor database. BMC Bioinformatics 8:42

Ricachenevsky FK, Sperotto RA, Menguer PK, Fett JP (2010) Identification of Feexcess-induced genes in rice shoots reveals a WRKY transcription factor responsive to Fe, drought and senescence. Mol Biol Rep 37(8):3735-3745

Ruijter JM, Ramakers C, Hoogaars WMH, Karlen Y, Bakker O, van den Hoff MJB, Moorman AFM (2009) Amplification efficiency: linking baseline and bias in the analysis of quantitative PCR data. Nucleic Acids Res 37(6)

Schmidt R, Schippers JHM, Mieulet D, Obata T, Fernie AR, Guiderdoni E, MuellerRoeber B (2013) MULTIPASS, a rice R2R3-type MYB transcription factor, regulates adaptive growth by integrating multiple hormonal pathways. Plant J 76(2):258-273

Septiningsih EM, Pamplona AM, Sanchez DL, Neeraja CN, Vergara GV, Heuer S, Ismail AM, Mackill DJ (2009) Development of submergence-tolerant rice cultivars: the Sub1 locus and beyond. Ann Bot 103(2):151-160

Septiningsih EM, Sanchez DL, Singh N, Sendon PM, Pamplona AM, Heuer S, Mackill DJ (2012) Identifying novel QTLs for submergence tolerance in rice cultivars IR72 and Madabaru. Theor Appl Genet 124(5):867-874

Shankar R, Bhattacharjee A, Jain M (2016) Transcriptome analysis in different rice cultivars provides novel insights into desiccation and salinity stress responses. Sci Rep 6:23719

Singh HP, Singh BB, Ram PC (2001) Submergence tolerance of rainfed lowland rice: search for physiological marker traits. J Plant Physiol 158(7):883-889

Singh N, Dang TT, Vergara GV, Pandey DM, Sanchez D, Neeraja CN, Septiningsih EM, Mendioro M, Tecson-Mendoza EM, Ismail AM, Mackill DJ, Heuer S (2010) 
Molecular marker survey and expression analyses of the rice submergencetolerance gene SUB1A. Theor Appl Genet 121(8):1441-1453

Singh P, Sinha AK (2016) A positive feedback loop governed by SUB1A1 interaction with MITOGEN-ACTIVATED PROTEIN KINASE3 imparts submergence tolerance in Rice. Plant Cell 28(5):1127-1143

Singh S, Mackill DJ, Ismail AM (2009) Responses of SUB1 rice introgression lines to submergence in the field: yield and grain quality. Field Crop Res 113(1):12-23

Skinner JS, von Zitzewitz J, Szucs P, Marquez-Cedillo L, Filichkin T, Amundsen K, Stockinger EJ, Thomashow MF, Chen THH, Hayes PM (2005) Structural, functional, and phylogenetic characterization of a large CBF gene family in barley. Plant Mol Biol 59(4):533-551

Smyth GK (2005) Limma: linear models for microarray data. In: Gentleman R, Carey V, Dudoit S, Irizarry R, Huber W (eds) Bioinformatics and computational Biology solutions using R and bioconductor. Springer, New York, pp 397-420

Sutoh K, Yamauchi D (2003) Two cis-acting elements necessary and sufficient for gibberellin-upregulated proteinase expression in rice seeds. Plant J 34(5):635-645

Suzuki M, Ketterling MG, McCarty DR (2005) Quantitative statistical analysis of cisregulatory sequences in $A B A N P 1$-and CBF/DREB1-regulated genes of Arabidopsis. Plant Physiol 139(1):437-447

Terzaghi WB, Cashmore AR (1995) Light-regulated transcription. Annu Rev Plant Physiol Plant Mol Biol 46:445-474

Todaka D, Nakashima K, Maruyama K, Kidokoro S, Osakabe Y, Ito Y, Matsukura S, Fujita Y, Yoshiwara K, Ohme-Takagi M, Kojima M, Sakakibara H, Shinozaki K, Yamaguchi-Shinozaki K (2012) Rice phytochrome-interacting factor-like protein OSPIL1 functions as a key regulator of internode elongation and induces a morphological response to drought stress. Proc Natl Acad Sci U S A 109(39):15947-15952

Toyofuku K, T-a U, Yamaguchi J (1998) Promoter elements required for sugarrepression of the RAmy3D gene for a-amylase in rice. FEBS Lett 428(3):275-280

Tremousaygue D, Garnier L, Bardet C, Dabos P, Herve C, Lescure B (2003) Internal telomeric repeats and 'TCP domain' protein-binding sites co-operate to regulate gene expression in Arabidopsis Thaliana cycling cells. Plant J 33(6):957-966

Vandepoele K, Vlieghe K, Florquin K, Hennig L, Beemster GTS, Gruissem W, Van De Peer Y, Inze D, De Veylder L (2005) Genome-wide identification of potential plant E2F target genes. Plant Physiol 139(1):316-328

Villain P, Mache R, Zhou DX (1996) The mechanism of GT element-mediated cell type-specific transcriptional control. J Biol Chem 271(51):32593-32598

Voesenek LACJ, Bailey-Serres J (2009) PLANT BIOLOGY genetics of high-rise rice. Nature 460(7258):959-960

Wang C, Zhang Q, Shou HX (2009) Identification and expression analysis of OsHsfs in rice. J Zhejiang Univ Sci B 10(4):291-300

Wang L, Ying YH, Narsai R, Ye LX, Zheng LQ, Tian JL, Whelan J, Shou HX (2013) Identification of OsbHLH133 as a regulator of iron distribution between roots and shoots in Oryza sativa. Plant Cell and Environment 36(1): 224-236.

Washida H, Wu CY, Suzuki A, Yamanouchi U, Akihama T, Harada K, Takaiwa F (1999) Identification of cis-regulatory elements required for endosperm expression of the rice storage protein glutelin gene GluB-1. Plant Mol Biol 40(1):1-12

Welchen E, Gonzalez DH (2005) Differential expression of the Arabidopsis cytochrome c genes Cytc-1 and Cytc-2. Evidence for the involvement of TCP-domain protein-binding elements in anther- and meristem-specific expression of the Cytc-1 gene. Plant Physiol 139(1):88-100

Welchen E, Gonzalez DH (2006) Overrepresentation of elements recognized by TCP-domain transcription factors in the upstream regions of nuclear genes encoding components of the mitochondrial oxidative phosphorylation machinery. Plant Physiol 141(2):540-545

Wu CY, Washida H, Onodera Y, Harada K, Takaiwa F (2000) Quantitative nature of the Prolamin-box, ACGT and AACA motifs in a rice glutelin gene promoter: minimal cis-element requirements for endosperm-specific gene expression. Plant J 23(3):415-421

Wu H, Ye H, Yao R, Zhang T, Xiong L (2015) OsJAZ9 acts as a transcriptional regulator in jasmonate signaling and modulates salt stress tolerance in rice. Plant Sci 232:1-12

Wu T, Yang C, Ding B, Feng Z, Wang Q, He J, Tong J, Xiao L, Jiang L, Wan J (2016) Microarray-based gene expression analysis of strong seed dormancy in rice cv. N22 and less dormant mutant derivatives. Plant Physiol Biochem 99:27-38

Xiang J, Wu H, Zhang Y, Zhang Y, Wang Y, Li Z, Lin H, Chen H, Zhang J, Zhu D (2017) Transcriptomic analysis of Gibberellin- and Paclobutrazol-treated Rice seedlings under submergence. Int J Mol Sci 18(10):2225
Xiong HY, Li JJ, Liu PL, Duan JZ, Zhao Y, Guo X, Li Y, Zhang HL, Ali J, Li ZC (2014) Overexpression of OsMYB48-1, a novel MYB-related transcription factor, enhances drought and salinity tolerance in Rice. PLoS One 9(3)

Xu K, Xu X, Fukao T, Canlas P, Maghirang-Rodriguez R, Heuer S, Ismail AM, BaileySerres J, Ronald PC, Mackill DJ (2006) Sub1A is an ethylene-response-factor-like gene that confers submergence tolerance to rice. Nature 442(7103):705-708

Xu KN, Deb R, Mackill DJ (2004) A microsatellite marker and a codominant PCRbased marker for marker-assisted selection of submergence tolerance in rice. Crop Sci 44(1):248-253

Yang YW, Chen HC, Jen WF, Liu LY, Chang MC (2015) Comparative Transcriptome analysis of shoots and roots of TNG67 and TCN1 Rice seedlings under cold stress and following subsequent recovery: insights into metabolic pathways, Phytohormones, and transcription factors. PLoS One 10(7):e0131391

Ye H, Du H, Tang N, Li X, Xiong L (2009) Identification and expression profiling analysis of TIFY family genes involved in stress and phytohormone responses in rice. Plant Mol Biol 71(3):291-305

Yuan L-B, Dai Y-S, Xie L-J, Yu L-J, Zhou Y, Lai Y-X, Yang Y-C, Xu L, Chen Q-F, Xiao $S$ (2017) Jasmonate regulates plant responses to Postsubmergence Reoxygenation through transcriptional activation of antioxidant synthesis. Plant Physiol 173(3):1864-1880

Zheng SJ (2010) Iron homeostasis and iron acquisition in plants: maintenance, functions and consequences (vol 105, pg 799, 2010). Ann Bot 105(6): 1071-1071.

Zhou DX (1999) Regulatory mechanism of plant gene transcription by GTelements and GT-factors. Trends Plant Sci 4(6):210-214

Zhu Y, Cai XL, Wang ZY, Hong MM (2003) An interaction between a MYC protein and an EREBP protein is involved in transcriptional regulation of the rice $\mathrm{Wx}$ gene. J Biol Chem 278(48):47803-47811

\section{Submit your manuscript to a SpringerOpen ${ }^{\circ}$ journal and benefit from:}

- Convenient online submission

- Rigorous peer review

- Open access: articles freely available online

- High visibility within the field

- Retaining the copyright to your article

Submit your next manuscript at springeropen.com 